\title{
On the Initial-Boundary-Value Problem for the Time-Fractional Diffusion Equation on the Real Positive Semiaxis
}

\author{
D. Goos, ${ }^{1,2}$ G. Reyero, ${ }^{1}$ S. Roscani, ${ }^{1,2}$ and E. Santillan Marcus ${ }^{1,3}$ \\ ${ }^{1}$ Departamento de Matemática, FCEIA, Universidad Nacional de Rosario, Pellegrini 250, S2000BTP Rosario, Argentina \\ ${ }^{2}$ CONICET, Departamento de Matemática, FCEIA, Universidad Nacional de Rosario, Pellegrini 250, S2000BTP Rosario, Argentina \\ ${ }^{3}$ Departamento de Matemática, FCE, Universidad Austral, Paraguay 1950, S2000FZF Rosario, Argentina
}

Correspondence should be addressed to S. Roscani; sabrinaroscani@gmail.com

Received 10 July 2015; Accepted 31 August 2015

Academic Editor: Nasser-Eddine Tatar

Copyright (C) 2015 D. Goos et al. This is an open access article distributed under the Creative Commons Attribution License, which permits unrestricted use, distribution, and reproduction in any medium, provided the original work is properly cited.

We consider the time-fractional derivative in the Caputo sense of order $\alpha \in(0,1)$. Taking into account the asymptotic behavior and the existence of bounds for the Mainardi and the Wright function in $\mathbb{R}^{+}$, two different initial-boundary-value problems for the time-fractional diffusion equation on the real positive semiaxis are solved. Moreover, the limit when $\alpha \nearrow 1$ of the respective solutions is analyzed, recovering the solutions of the classical boundary-value problems when $\alpha=1$, and the fractional diffusion equation becomes the heat equation.

\section{Introduction}

The one-dimensional heat equation has become the paradigm for the all-embracing study of parabolic partial differential equations, linear and nonlinear. A methodical development of a variety of aspects of this paradigm can be seen in [1-3].

This paper deals with two problems associated with the time-fractional diffusion equation, obtained from the standard heat equation by replacing the first-order timederivative by a fractional derivative of order $\alpha>0$ in the Caputo sense:

$$
\begin{aligned}
& { }_{0} D_{t}^{\alpha} u(x, t)=\lambda^{2} u_{x x}(x, t), \\
& 0<x<\infty, 0<t<T, 0<\alpha<1,
\end{aligned}
$$

where the fractional derivative in the Caputo sense of arbitrary order $\alpha>0$ is given by

$$
\begin{aligned}
& { }_{a} D^{\alpha} f(t) \\
& = \begin{cases}\frac{1}{\Gamma(n-\alpha)} \int_{a}^{t}(t-\tau)^{n-\alpha-1} f^{(n)}(\tau) d \tau, & n-1<\alpha<n \\
f^{(n)}(t), & \alpha=n,\end{cases}
\end{aligned}
$$

where $n \in \mathbb{N}$ and $\Gamma$ is the Gamma function defined by $\Gamma(x)=$ $\int_{0}^{\infty} w^{x-1} e^{-w} d w$.
The interest on (1) has been in constant increase during the last 30 years. So many authors have studied it [4-10] and, among the several applications that have been studied, Mainardi [11] focused on the application to the theory of linear viscoelasticity.

A comprehensive analysis of the Cauchy problem associated with this equation can be found in [12] and a physical meaning is discussed in [13].

The two initial-boundary-value problems considered are

$$
\begin{aligned}
&{ }_{0} D_{t}^{\alpha} c(x, t)=\lambda^{2} \frac{\partial^{2} c}{\partial x^{2}}(x, t) \\
& 0<x<\infty, 0<t<T, 0<\alpha<1, \\
& c(x, 0)=f(x) \quad 0<x<\infty, \\
& c(0, t)=g(t) \quad 0<t<T, \\
&{ }_{0} D_{t}^{\alpha} c(x, t)=\lambda^{2} \frac{\partial^{2} c}{\partial x^{2}}(x, t) \\
& \quad 0<x<\infty, 0<t<T, 0<\alpha<1, \\
& c(x, 0)=f(x) \quad 0<x<\infty, \\
& \frac{\partial c}{\partial x}(0, t)=g(t) \quad 0<t<T,
\end{aligned}
$$


associated with the Dirichlet and Newmann boundary conditions, respectively.

Some variants of problem (3) have already been solved. In [10], using the Mellin transform,

$$
\begin{aligned}
& T\left(t^{-\alpha / 2} x\right)=\pi^{-1 / 2} h\left(\frac{1}{2 a t^{-\alpha / 2} x}\right), \\
& \text { where } h(z)=H_{23}^{21}\left(z \mid \begin{array}{l}
(1,1) ;(1, \lambda / 2) \\
(1 / 2,1 / 2) ;(1,1 / 2) ;(0,1)
\end{array}\right)
\end{aligned}
$$

is the Fox function of the given parameters, is obtained as a solution to the particular problem

$$
\begin{aligned}
{ }_{0} D_{t}^{\alpha} T(x, t) & =a \frac{\partial^{2} T}{\partial x^{2}}(x, t) \\
& 0<x<\infty, a>0,0<t<T, 0<\alpha<2, \\
T(x, 0) & =1, \quad 0<x<\infty \\
T(0, t) & =0, \quad 0<t<T .
\end{aligned}
$$

In [14] the two problems for the time-fractional diffusion equation are considered in two disjoint intervals for the spatial variable which cover the set $0<x<\infty$. Here the following conditions are imposed:

$$
\begin{aligned}
{ }_{0} D_{t}^{\alpha} T_{1}(x, t) & =a_{1} \frac{\partial^{2} T_{1}}{\partial x^{2}}(x, t) \\
& 0<x<L, 0<t<T, 0<a_{1}, 0<\alpha \leq 2 \\
T_{1}(x, 0) & =T_{0}, \quad 0<x<L, 0<\alpha \leq 2 \\
\frac{\partial T_{1}}{\partial t}(x, 0) & =0, \quad 0<x<L, \quad 1<\alpha \leq 2, \\
{ }_{0} D_{t}^{\beta} T_{2}(x, t) & =a_{2} \frac{\partial^{2} T_{2}}{\partial x^{2}}(x, t) \\
L & <x<\infty, 0<t<T, 0<a_{2}, 0<\beta \leq 2 \\
T_{2}(x, 0) & =0, \quad L<x<\infty, 0<\beta \leq 2 \\
\frac{\partial T_{2}}{\partial t}(x, 0) & =0, \quad L<x<\infty, 1<\beta \leq 2,
\end{aligned}
$$

and for the particular case $\alpha=\beta$, which is the case of our interest, the solutions

$$
\begin{aligned}
& T_{1}(x, t) \cong T_{0}-\frac{T_{0}}{1+\gamma} \mathscr{W}\left(-\frac{L-x}{\sqrt{a_{1}} \tau^{\alpha / 2}} ;-\frac{\alpha}{2} ; 1\right), \\
& T_{2}(x, t) \cong T \frac{T_{0} \gamma}{1+\gamma} \mathscr{W}\left(-\frac{x-L}{\sqrt{a_{2}} \tau^{\alpha / 2}} ;-\frac{\alpha}{2} ; 1\right)
\end{aligned}
$$

are presented, where $\gamma$ is a particular constant of the problem and $\mathscr{W}$ is the Wright function, which will be defined in the next section.

In both cases no complete mathematical proof that the obtained functions actually are solutions of the fractionaldiffusion equation is presented. We propose here a different approach involving convolutions that allows us to achieve more general solutions to problem (3) and we also solve problem (4) for the Neumann boundary condition. Moreover, we provide in each case a rigorous proof that the proposed function is a solution of the considered problem. Finally we show how from given solutions to the fractional diffusion equation one can construct new ones that verify different boundary conditions.

The paper is presented as follows. Some useful properties about the behavior of Wright functions are given in Section 2. In Sections 3, 4, and 5 the two problems enunciated previously will be solved. At the end of Sections 3 and 5 the limit when $\alpha \nearrow 1$ of the respective solutions will be done, recovering the respective solutions of the classical boundary-value problems when $\alpha=1$ and (1) becomes the heat equation.

\section{Preliminaries: Some Results about the Special Functions Involved}

Definition 1. For every $z \in \mathbb{C}, \alpha>-1$, and $\beta \in \mathbb{R}$ the Wright function is defined by

$$
\mathscr{W}(z ; \alpha ; \beta)=\sum_{k=0}^{\infty} \frac{z^{k}}{k ! \Gamma(\alpha k+\beta)} .
$$

Definition 2. For every $z \in \mathbb{C}, 0<v<1$ the Mainardi function is defined by

$$
\mathscr{M}_{\nu}(z)=\mathscr{W}(-z ;-v ; 1-v)=\sum_{k=0}^{\infty} \frac{(-z)^{k}}{k ! \Gamma(-v k+1-\nu)} .
$$

Note 1. This series are absolutely convergent over compact sets and so its derivatives are easy to calculate:

$$
\begin{aligned}
\frac{d}{d z} \mathscr{W}(z, \alpha, \beta) & =\sum_{n=0}^{\infty} \frac{d}{d z} \frac{(z)^{n}}{n ! \Gamma(\alpha n+\beta)} \\
& =\sum_{n=1}^{\infty} \frac{(z)^{n-1}}{(n-1) ! \Gamma(\alpha n+\beta)} \\
& =\sum_{n=0}^{\infty} \frac{(z)^{n}}{n ! \Gamma(\alpha(n+1)+\beta)} \\
& =\sum_{n=0}^{\infty} \frac{(z)^{n}}{n ! \Gamma(\alpha n+(\alpha+\beta))} \\
& =\mathscr{W}(z, \alpha, \alpha+\beta) .
\end{aligned}
$$

For the special case of the Mainardi function, we have

$$
\frac{d}{d z} \mathscr{M}_{\nu}(z)=-\mathscr{W}(-z ;-v ; 1-2 v)
$$

2.1. Asymptotic Behavior. The following asymptotic behavior for the Mainardi function was proved in [15]:

$$
\mathscr{M}_{\nu}\left(\frac{x}{\nu}\right) \sim a(\nu) x^{(\nu-1 / 2) /(1-\nu)} \exp \left[-b(\nu) x^{1 /(1-\nu)}\right],
$$

where $a(\nu)=1 / \sqrt{2 \pi(1-\nu)}>0, b(\nu)=(1-\nu) / \nu$. 
Theorem 3. If $-1<\rho<0, y=-z$, and $|\arg y| \leq$ $\min \{(3 / 2) \pi(1+\rho), \pi\}-\epsilon, \epsilon>0$, then

$$
\mathscr{W}(z, \rho, \beta)=I(Y), \quad Y \longrightarrow \infty
$$

where

$$
\begin{aligned}
I(Y) & =Y^{1 / 2-\beta} e^{-Y}\left\{\sum_{m=0}^{M-1} A_{m} Y^{-m}+O\left(Y^{-M}\right)\right\}, \\
Y & =(1+\rho)\left((-\rho)^{-\rho} y\right)^{1 /(1+\rho)} .
\end{aligned}
$$

The coefficients $A_{m}, m=0,1, \ldots$ are defined by the asymptotic expansion

$$
\begin{gathered}
\frac{\Gamma(1-\beta-\rho t)}{2 \pi(-\rho)^{-\rho t}(1+\rho)^{(1+\rho)(t+1)} \Gamma(t+1)} \\
=\sum_{m=0}^{M-1} \frac{(-1)^{m} A_{m}}{\Gamma((1+\rho) t+\beta+1 / 2+m)} \\
\quad+O\left(\frac{1}{\Gamma((1+\rho) t+\beta+1 / 2+M)}\right),
\end{gathered}
$$

valid for $\arg t, \arg (-\rho t)$, and $\arg (1-\beta-\rho t)$ all lying between $-\pi$ and $\pi$ and tending to infinity.

This theorem was proved in [16]. The next results follow.

Corollary 4. The following limits hold

$$
\begin{aligned}
\lim _{x \rightarrow \infty} \mathscr{W}\left(-x,-\frac{\alpha}{2}, 1\right) & =0, \\
\lim _{x \rightarrow \infty} \mathscr{W}\left(-x,-\frac{\alpha}{2}, 1+\frac{\alpha}{2}\right) & =0 .
\end{aligned}
$$

Corollary 5. The next limit holds

$$
\lim _{x \rightarrow \infty} \mathscr{M}_{\alpha / 2}(x)=0
$$

Corollary 6. If $0<\alpha<1$ and $x \in \mathbb{R}^{+}$, there exists $R>0$ such that

$$
\begin{aligned}
& \left|\mathscr{W}\left(-x,-\frac{\alpha}{2}, 1-\alpha\right)\right| \\
& \quad<P\left(b x^{1 /(1-\alpha / 2)}\right) \exp \left\{-b x^{1 /(1-\alpha / 2)}\right\} \quad \forall x>R,
\end{aligned}
$$

where $P(x)$ is a polynomial function of degree less than or equal to 1 and $b=(1-\alpha / 2)(\alpha / 2)^{\alpha /(2-\alpha)}>0$.

Proof. Let us consider the function $\mathscr{W}(-x,-\alpha / 2,1-\alpha)$. One has

$$
z=-x \Longrightarrow y=x, \quad \arg y=0
$$

Taking

$$
\begin{aligned}
& Y=\left(1-\frac{\alpha}{2}\right)\left(\left(\frac{\alpha}{2}\right)^{\alpha / 2} x\right)^{1 /(1-\alpha / 2)}=b x^{1 /(1-\alpha / 2)}, \\
& b=\left(1-\frac{\alpha}{2}\right)\left(\frac{\alpha}{2}\right)^{\alpha /(2-\alpha)}>0,
\end{aligned}
$$

Theorem 3 gives the equation

$$
\begin{aligned}
& \mathscr{W}\left(-x,-\frac{\alpha}{2}, 1-\alpha\right)=\left(b x^{1 /(1-\alpha / 2)}\right)^{\alpha-1 / 2} \\
& \cdot \exp \left\{-b x^{1 /(1-\alpha / 2)}\right\}\left\{\sum_{m=0}^{M-1} A_{m}\left(b x^{1 /(1-\alpha / 2)}\right)^{-m}\right. \\
& \left.+O\left(\left(b x^{1 /(1-\alpha / 2)}\right)^{-M}\right)\right\} .
\end{aligned}
$$

Or equivalently,

$$
\begin{gathered}
\frac{\mathscr{W}(-x,-\alpha / 2,1-\alpha)}{\left(b x^{1 /(1-\alpha / 2)}\right)^{\alpha-1 / 2} \exp \left\{-b x^{1 /(1-\alpha / 2)}\right\}} \\
-\sum_{m=0}^{M-1} A_{m}\left(b x^{1 /(1-\alpha / 2)}\right)^{-m} \\
=O\left(\left(b x^{1 /(1-\alpha / 2)}\right)^{-M}\right) .
\end{gathered}
$$

Taking $M=1$, there exists $R>0$ such that

$$
\left|\frac{\left(\mathscr{W}(-x,-\alpha / 2,1-\alpha) /\left(b x^{1 /(1-\alpha / 2)}\right)^{\alpha-1 / 2} \exp \left\{-b x^{1 /(1-\alpha / 2)}\right\}\right)-A_{0}}{\left(b x^{1 /(1-\alpha / 2)}\right)^{-1}}\right| \leq C \quad \text { if } x>R
$$

Then

$$
\begin{aligned}
\left|\mathscr{W}\left(-x,-\frac{\alpha}{2}, 1-\alpha\right)\right| \leq\left(b x^{1 /(1-\alpha / 2)}\right)^{\alpha-1 / 2} \\
\quad \cdot\left(C\left(b x^{1 /(1-\alpha / 2)}\right)^{-1}+\left|A_{0}\right|\right) \exp \left\{-b x^{1 /(1-\alpha / 2)}\right\},
\end{aligned}
$$$$
\text { if } x>R \text {. }
$$

If $0<\alpha<1 / 2,\left(b x^{1 /(1-\alpha / 2)}\right)^{\alpha-1 / 2}<1 /\left(b R^{1 /(1-\alpha / 2)}\right)^{1 / 2-\alpha}$. If $\alpha=1 / 2,\left(b x^{1 /(1-\alpha / 2)}\right)^{\alpha-1 / 2}=1$. If $1 / 2<\alpha<1$, taking $R$ large enough so that $b x^{1 /(1-\alpha / 2)}>1$ if $x>R$, it follows that

$$
\left(b x^{1 /(1-\alpha / 2)}\right)^{\alpha-1 / 2}<\left(b x^{1 /(1-\alpha / 2)}\right) .
$$


Hence there exists two constants $B_{0}$ and $B_{1}$ depending on $\alpha$ such that

$$
\left(b x^{1 /(1-\alpha / 2)}\right)^{\alpha-1 / 2}<B_{0}+B_{1}\left(b x^{1 /(1-\alpha / 2)}\right) .
$$

Finally,

$$
\begin{aligned}
& \left(b x^{1 /(1-\alpha / 2)}\right)^{\alpha-1 / 2}\left(C\left(b x^{1 /(1-\alpha / 2)}\right)^{-1}+\left|A_{0}\right|\right) \\
& <\left(B_{0}+B_{1}\left(b x^{1 /(1-\alpha / 2)}\right)\right)\left(C\left(b x^{1 /(1-\alpha / 2)}\right)^{-1}+\left|A_{0}\right|\right) \\
& =P_{1}\left(\left(b x^{1 /(1-\alpha / 2)}\right)^{-1}\right)+P_{2}\left(b x^{1 /(1-\alpha / 2)}\right) \\
& \leq \widetilde{C}+P_{2}\left(b x^{1 /(1-\alpha / 2)}\right)=P\left(b x^{1 /(1-\alpha / 2)}\right)
\end{aligned}
$$

where $P$ is a polynomial function of degree less than or equal to 1 .

Therefore

$$
\begin{aligned}
& \left|\mathscr{W}\left(-x,-\frac{\alpha}{2}, 1-\alpha\right)\right| \\
& \quad<P\left(b x^{1 /(1-\alpha / 2)}\right) \exp \left\{-b x^{1 /(1-\alpha / 2)}\right\}, \quad \text { if } x>R .
\end{aligned}
$$

Corollary 7. If $0<\alpha<1$ and $x \in \mathbb{R}^{+}$, there exists $R>0$ such that

$$
\begin{aligned}
&\left|\mathscr{W}\left(-x,-\frac{\alpha}{2}, 1\right)\right|<K e^{-b x} \\
& \forall x>R \text { where } b=\left(1-\frac{\alpha}{2}\right)\left(\frac{\alpha}{2}\right)^{\alpha /(2-\alpha)} .
\end{aligned}
$$

2.2. Some Bounds and Convergence. The assertions in this subsection were proved in [17].

Lemma 8. If $0<\alpha<1, \mathscr{M}_{\alpha / 2}(x)$ is a strictly decreasing positive function in $\mathbb{R}^{+}$.

Corollary 9. If $x>0, \mathscr{M}_{\alpha / 2}(x)<1 / \Gamma(1-\alpha / 2)$.

Corollary 10. If $0<\alpha<1, \mathscr{W}(-x,-\alpha / 2,1)$ is a positive and decreasing function in $\mathbb{R}^{+}$such that

$$
0<\mathscr{W}\left(-x,-\frac{\alpha}{2}, 1\right) \leq 1, \quad \forall x \in \mathbb{R}_{0}^{+} .
$$

Note 2. Note that

$$
\begin{aligned}
\mathscr{W}\left(-x,-\frac{1}{2}, 1\right) & =\int_{\infty}^{x}\left(\frac{\partial}{\partial x} \mathscr{W}\left(-\xi,-\frac{1}{2}, 1\right)\right) d \xi \\
& =\int_{\infty}^{x}-\mathscr{W}\left(-\xi,-\frac{1}{2}, \frac{1}{2}\right) d \xi \\
& =\int_{x}^{\infty} \mathscr{W}\left(-\xi,-\frac{1}{2}, \frac{1}{2}\right) d \xi \\
& =\int_{x}^{\infty} \frac{1}{\sqrt{\pi}} e^{-\xi^{2} / 4} d \xi \\
& =\frac{2}{\sqrt{\pi}} \int_{x / 2}^{\infty} \frac{1}{\sqrt{\pi}} e^{-\xi^{2}} d \xi=\operatorname{erfc}\left(\frac{x}{2}\right) .
\end{aligned}
$$

Hence

$$
1-\mathscr{W}\left(-x,-\frac{1}{2}, 1\right)=\operatorname{erf}\left(\frac{x}{2}\right) .
$$

Lemma 11. If $x \in \mathbb{R}_{0}^{+}$and $\alpha \in(0,1)$,

(1) $\lim _{\alpha>1} \mathscr{M}_{\alpha / 2}(x)=\mathscr{M}_{1 / 2}(x)=e^{-x^{2} / 4} / \sqrt{\pi}$,

(2) $\lim _{\alpha>1}[1-\mathscr{W}(-x,-\alpha / 2,1)]=(1 / \sqrt{\pi}) \operatorname{erf}(x / 2)$.

\section{Solving the Initial-Boundary-Value Problem for the Time-Fractional Diffusion Equation on the Real Positive Semiaxis with Temperature-Boundary Condition}

Let us consider problem (3). The principle of superposition is valid due to the linearity of the Caputo derivative. Then, solving problem (3) is equivalent to solving the two auxiliary problems:

$$
\begin{aligned}
{ }_{0} D_{t}^{\alpha} c_{1}(x, t) & =\lambda^{2} \frac{\partial^{2} c_{1}}{\partial x^{2}}(x, t) \\
& 0<x<\infty, 0<t<T, 0<\alpha<1, \\
c_{1}(x, 0) & =f(x) \quad 0<x<\infty, \\
c_{1}(0, t) & =0 \quad 0<t<T, \\
{ }_{0} D_{t}^{\alpha} c_{2}(x, t) & =\lambda^{2} \frac{\partial^{2} c_{2}}{\partial x^{2}}(x, t) \\
\quad 0<x<\infty, 0<t<T, 0<\alpha<1, & 0<x<\infty, \\
c_{2}(x, 0) & =0 \quad 0<t<T . \\
c_{2}(0, t) & =g(t) \quad 0<1 .
\end{aligned}
$$

Problem (34) was solved in [18] and its solution is given by

$$
\begin{aligned}
& c_{1}(x, t)=\int_{0}^{\infty} \frac{1}{2 \lambda t^{\alpha / 2}}\left[\mathscr{M}_{\alpha / 2}\left(\frac{|x-\xi|}{t^{\alpha / 2}}\right)\right. \\
& \left.-\mathscr{M}_{\alpha / 2}\left(\frac{|x+\xi|}{t^{\alpha / 2}}\right)\right] f(\xi) d \xi,
\end{aligned}
$$

where the function $\mathscr{M}_{\alpha / 2}(\cdot)$ is the Mainardi function defined in (10) and $f$ is a continuous bounded function in $\mathbb{R}_{0}^{+}$(which guarantees that $c_{1}$ is a solution; see the Cauchy problem in [19]).

In [17] it was proved that

$$
z(x, t)=A+(B-A) \mathscr{W}\left(\frac{-x}{\lambda t^{\alpha / 2}},-\frac{\alpha}{2}, 1\right),
$$

where $\mathscr{W}(\cdot,-\alpha / 2,1)$ is the Wright function of parameters $-\alpha / 2$ and 1 defined in (9), is a solution to the problem

$$
\begin{aligned}
{ }_{0} D_{t}^{\alpha} z(x, t)= & \lambda^{2} \frac{\partial^{2} z}{\partial x^{2}}(x, t) \\
& 0<x<\infty, 0<t<T, 0<\alpha<1, \\
z(x, 0) & =A \quad 0<x<\infty, \\
z(0, t) & =B \quad 0<t<T .
\end{aligned}
$$


Then, we can assure that

$$
v(x, t)=\mathscr{W}\left(\frac{-x}{\lambda t^{\alpha / 2}},-\frac{\alpha}{2}, 1\right)
$$

is a solution to problem

$$
\begin{array}{rl}
{ }_{0} D_{t}^{\alpha} v(x, t)=\lambda^{2} \frac{\partial^{2} v}{\partial x^{2}}(x, t) & \\
& 0<x<\infty, 0<t<T, 0<\alpha<1, \\
v(x, 0)=0 & 0<x<\infty, \\
v(0, t)=1 & 0<t<T .
\end{array}
$$

Taking into account Note 1 and Corollary 5 , function $v$ can be expressed as

$$
\begin{aligned}
v(x, t) & =\int_{0}^{t} \frac{\partial}{\partial \tau} \mathscr{W}\left(\frac{-x}{\lambda \tau^{\alpha / 2}},-\frac{\alpha}{2}, 1\right) d \tau \\
& =\int_{0}^{t} \mathscr{M}_{\alpha / 2}\left(\frac{x}{\lambda \tau^{\alpha / 2}}\right) \frac{\alpha x}{2 \lambda \tau^{\alpha / 2+1}} d \tau .
\end{aligned}
$$

Let

$$
\begin{aligned}
K(x, t) & =\mathscr{M}_{\alpha / 2}\left(\frac{x}{\lambda t^{\alpha / 2}}\right) \frac{\alpha x}{2 \lambda t^{\alpha / 2+1}}, \\
\mathbf{1}_{\left[0, t_{0}\right]}(t) & = \begin{cases}1 & \text { if } 0<t<t_{0} \\
0 & \text { else. }\end{cases}
\end{aligned}
$$

Then function $v$ can be written as a convolution in the $t-$ variable:

$$
v(x, t)=K(x, t) * \mathbf{1}_{[0, t]} .
$$

This new way of expressing $v$ leads us to propose the following function:

$$
\begin{aligned}
& c_{2}(x, t)=K(x, t) * g(t) \mathbf{1}_{[0, t]} \\
& \quad=\int_{0}^{t} \mathscr{M}_{\alpha / 2}\left(\frac{x}{\lambda(t-\tau)^{\alpha / 2}}\right) \frac{\alpha x}{2 \lambda(t-\tau)^{\alpha / 2+1}} g(\tau) d \tau
\end{aligned}
$$

as a solution to problem (35).

In order to prove this assertion, let us enunciate the following lemma.

Lemma 12. Let $\mathscr{K}(t-\tau) f(\tau)$ be a function that verifies the following conditions:

$\mathscr{K}(t-\tau) f(\tau)$ is a $\tau$-integrable function in $[0, t]$,

$$
\left|\frac{\partial}{\partial t} \mathscr{K}(t-\tau) f(\tau)\right| \leq j(\tau) \in L^{1}[0, t],
$$

$\left|\frac{\partial}{\partial \eta} \mathscr{K}(\eta-\tau) \frac{f(\tau)}{(t-\eta)^{\alpha}}\right| \in L^{1}(\Omega)$,

where $\Omega=\left\{(\eta, \tau) \in \mathbb{R}^{2}: \eta \in(0, t), 0 \leq \tau \leq \eta\right\}$

$\lim _{\tau / \eta} \mathscr{K}(\eta-\tau) f(\tau)=h(\eta) \in L^{1}(0, t)$.
Then

$$
\begin{aligned}
{ }_{0} D_{t}^{\alpha} & \left(\int_{0}^{t} \mathscr{K}(t-\tau) f(\tau) d \tau\right) \\
\quad= & \int_{0}^{t}\left({ }_{0} D_{t}^{\alpha} K(t-\tau)\right) f(\tau) d \tau+{ }_{0} I_{t}^{1-\alpha}(h(t)),
\end{aligned}
$$

where ${ }_{0} I^{1-\alpha}$ is the fractional integral of Riemann-Liouville of order $1-\alpha$ defined by

$$
{ }_{0} I^{1-\alpha} h(t)=\frac{1}{\Gamma(1-\alpha)} \int_{0}^{t}(t-\tau)^{(1-\alpha)-1} h(\tau) d \tau .
$$

Proof. Due to (46) and (48)

$$
\begin{aligned}
& \frac{d}{d t} \int_{0}^{t} \mathscr{K}(t-\tau) f(\tau) d \tau \\
& \quad=\int_{0}^{t} \frac{\partial}{\partial t} \mathscr{K}(t-\tau) f(\tau) d \tau+\lim _{\tau / t} \mathscr{K}(t-\tau) f(\tau) .
\end{aligned}
$$

Now

$$
\begin{aligned}
& { }_{0} D_{t}^{\alpha}\left(\int_{0}^{t} \mathscr{K}(t-\tau) f(\tau) d \tau\right)=\frac{1}{\Gamma(1-\alpha)} \\
& \cdot \int_{0}^{t} \frac{(\partial / \partial \eta) \int_{0}^{\eta} \mathscr{K}(\eta-\tau) f(\tau) d \tau}{(t-\eta)^{\alpha}} d \eta=\frac{1}{\Gamma(1-\alpha)} \\
& \cdot \int_{0}^{t} \frac{1}{(t-\eta)^{\alpha}}\left[\int_{0}^{\eta} \frac{\partial}{\partial \eta} \mathscr{K}(\eta-\tau) f(\tau) d \tau\right. \\
& \left.\quad+\lim _{\tau / \eta} \mathscr{K}(\eta-\tau) f(\tau)\right] d \eta .
\end{aligned}
$$

Since (47) holds, (52) is equal to

$$
\begin{aligned}
& \int_{0}^{t} \frac{f(\tau)}{\Gamma(1-\alpha)} \int_{\tau}^{t} \frac{(\partial / \partial \eta) K(\eta-\tau)}{(t-\eta)^{\alpha}} d \eta d \tau \\
& \quad+\frac{1}{\Gamma(1-\alpha)} \int_{0}^{t} \frac{\lim _{\tau / \eta} \mathscr{K}(\eta-\tau) f(\tau)}{(t-\eta)^{\alpha}} d \eta
\end{aligned}
$$

Substituting $s=\eta-\tau$,

$$
\begin{aligned}
& \frac{1}{\Gamma(1-\alpha)} \int_{\tau}^{t} \frac{(\partial / \partial \eta) \mathscr{K}(\eta-\tau)}{(t-\eta)^{\alpha}} d \eta \\
& \quad=\frac{1}{\Gamma(1-\alpha)} \int_{0}^{t-\tau} \frac{(\partial / \partial s) \mathscr{K}(s)}{(t-\tau-s)^{\alpha}} d s={ }_{0} D_{t}^{\alpha} K(t-\tau) .
\end{aligned}
$$

On the other hand,

$$
\begin{aligned}
& \frac{1}{\Gamma(1-\alpha)} \int_{0}^{t} \frac{\lim _{\tau>\eta} \mathscr{K}(\eta-\tau) f(\tau)}{(t-\eta)^{\alpha}} d \eta \\
& =\frac{1}{\Gamma(1-\alpha)} \int_{0}^{t} \frac{h(\eta)}{(t-\eta)^{\alpha}} d \eta={ }_{0} I_{t}^{1-\alpha}(h(t)) .
\end{aligned}
$$


Hence

$$
\begin{aligned}
{ }_{0} D_{t}^{\alpha} & \left(\int_{0}^{t} \mathscr{K}(t-\tau) f(\tau) d \tau\right) \\
& =\int_{0}^{t}\left({ }_{0} D_{t}^{\alpha} \mathscr{K}(t-\tau)\right) f(\tau) d \tau+{ }_{0} I_{t}^{1-\alpha}(h(t)) .
\end{aligned}
$$

Now the purpose is to prove that the kernel

$$
\mathscr{M}_{\alpha / 2}\left(\frac{x}{\lambda(t-\tau)^{\alpha / 2}}\right) \frac{\alpha x}{2 \lambda(t-\tau)^{\alpha / 2+1}} g(\tau)
$$

verifies the hypothesis of Lemma 12.

(i) Hypothesis (45). Consider the integral

$$
\begin{gathered}
\int_{0}^{t}\left|\mathscr{M}_{\alpha / 2}\left(\frac{x}{\lambda(t-\tau)^{\alpha / 2}}\right) \frac{\alpha x}{2 \lambda(t-\tau)^{\alpha / 2+1}} g(\tau)\right| d \tau \\
=\int_{x / \lambda t^{\alpha / 2}}^{\infty} \mathscr{M}_{\alpha / 2}(y)\left|g\left(t-\left(\frac{x}{\lambda y}\right)^{2 / \alpha}\right)\right| d y .
\end{gathered}
$$

We know that

$$
\int_{0}^{\infty} y^{n} \mathscr{M}_{\alpha / 2}(y) d y=\frac{\Gamma(n+1)}{\Gamma((\alpha / 2) n+1)} \quad \forall \alpha \in(0,2)
$$

(see [20]).

The Mainardi function is a positive and decreasing function in $\mathbb{R}^{+}$. (see [17]).

$g$ is a bounded function in $[0, t]$; that is,

$$
|g(\tau)| \leq M \quad \forall \tau \in[0, t]
$$

Then (58) is convergent and (57) is $\tau$-integrable in $[0, t]$.

(ii) Hypothesis (46). Consider the kernel

$$
\begin{aligned}
\frac{\partial}{\partial t}[ & \left.\mathscr{M}_{\alpha / 2}\left(\frac{x}{\lambda(t-\tau)^{\alpha / 2}}\right) \frac{\alpha x}{2 \lambda(t-\tau)^{\alpha / 2+1}}\right] g(\tau) \\
& =\mathscr{W}\left(-\frac{x}{\lambda(t-\tau)^{\alpha / 2}},-\frac{\alpha}{2}, 1-\alpha\right) \\
& \cdot\left(\frac{\alpha x}{2 \lambda(t-\tau)^{\alpha / 2+1}}\right)^{2} g(\tau)-\mathscr{M}_{\alpha / 2}\left(\frac{x}{\lambda(t-\tau)^{\alpha / 2}}\right) \\
& \cdot \frac{(\alpha / 2+1) \alpha x}{2 \lambda(t-\tau)^{\alpha / 2+2}} g(\tau) .
\end{aligned}
$$

Applying Corollary 6, there exists $\delta>0$ such that, for all $\tau \in$ $(t-\delta, t)$,

$$
\begin{gathered}
\mid \mathscr{W}\left(-\frac{x}{\lambda(t-\tau)^{\alpha / 2}},-\frac{\alpha}{2}, 1-\alpha\right)\left(\frac{\alpha x}{2 \lambda(t-\tau)^{\alpha / 2+1}}\right)^{2} \\
\cdot g(\tau) \mid \leq\left(c+d \frac{x}{\lambda(t-\tau)^{\alpha / 2}}\right)
\end{gathered}
$$

$$
\begin{aligned}
& \cdot \exp \left\{-b\left(\frac{x}{\lambda(t-\tau)^{\alpha / 2}}\right)^{2 /(2-\alpha)}\right\} \\
& \cdot\left(\frac{\alpha x}{2 \lambda(t-\tau)^{\alpha / 2+1}}\right)^{2}|g(\tau)|,
\end{aligned}
$$

$b>0, c, d$ constants.

And this is an integrable function; in fact, making the substitution

$$
\frac{x}{\lambda(t-\tau)^{\alpha / 2}}=r
$$

and considering the inequality

$$
\exp \{-x\} \leq \frac{n !}{x^{n}}, \quad \forall n \in \mathbb{N}, \forall x>0
$$

it yields that

$$
\begin{aligned}
& \int_{t-\delta}^{t}\left(c+d \frac{x}{\lambda(t-\tau)^{\alpha / 2}}\right) \\
& \cdot \exp \left\{-b\left(\frac{x}{\lambda(t-\tau)^{\alpha / 2}}\right)^{2 / 2-\alpha}\right\} \\
& \cdot\left(\frac{\alpha x}{2 \lambda(t-\tau)^{\alpha / 2+1}}\right)^{2}|g(\tau)| d \tau=\int_{x / \lambda \delta^{\alpha / 2}}^{\infty}(c+d r) \\
& \cdot \exp \left\{-b r^{2 / 2-\alpha}\right\} \frac{\alpha}{2} r\left(\frac{r \lambda}{x}\right)^{2 / \alpha}\left|g\left(t-\left(\frac{x}{\lambda r}\right)^{2 / \alpha}\right)\right| d r \\
& \leq C_{x, \lambda, \alpha} M \int_{x / \lambda \delta^{\alpha / 2}}^{\infty}(c+d r) r^{1+2 / \alpha} \\
& \cdot \exp \left\{-b r^{2 / 2-\alpha}\right\} d r \\
& \leq C_{x, \lambda, \alpha} M \int_{x / \lambda \delta^{\alpha / 2}}^{\infty} \frac{(c+d r) r^{1+2 / \alpha}}{b^{n} r^{2 n / 2-\alpha}} n ! d r .
\end{aligned}
$$

It it easy to see that for any $\alpha \in(0,1)$, there exists $n \in \mathbb{N}$ such that (65) is convergent. For example, if $\alpha=1 / 4$ we can take $n=10$.

Then, the first term of the sum (61) is bounded by an integrable function.

Let us consider the second term of sum (61). Making the substitution (63) and taking into account that the Mainardi function is a positive function, we have

$$
\begin{aligned}
& \int_{0}^{t}\left|\mathscr{M}_{\alpha / 2}\left(\frac{x}{\lambda(t-\tau)^{\alpha / 2}}\right) \frac{(\alpha / 2+1) \alpha x}{2 \lambda(t-\tau)^{\alpha / 2+2}} g(\tau)\right| d \tau \\
& \quad \leq M \int_{x / \lambda t^{\alpha / 2}}^{\infty}\left(\frac{\alpha}{2}+1\right) \mathscr{M}_{\alpha / 2}(r)\left(\frac{\lambda r}{x}\right)^{2 / \alpha} d r \\
& \quad \leq M C_{x, \lambda, \alpha} \int_{0}^{\infty} \mathscr{M}_{\alpha / 2}(r) r^{2 / \alpha} d r .
\end{aligned}
$$


Now, for any $\alpha \in(0,1)$, there exists $k \in \mathbb{N}$ such that $1 / k<$ $\alpha / 2<1$. Then, using (59), it yields

$$
\begin{aligned}
& M C_{x, \lambda, \alpha} \int_{0}^{\infty} \mathscr{M}_{\alpha / 2}(r) r^{2 / \alpha} d r \\
& \quad \leq M C_{x, \lambda, \alpha} \int_{0}^{\infty} \mathscr{M}_{\alpha / 2}(r) r^{k} d r \\
& \quad=M C_{x, \lambda, \alpha} \frac{\Gamma(k+1)}{\Gamma((\alpha / 2) k+1)} .
\end{aligned}
$$

(iii) Hypothesis (47). We have to prove that

$$
\begin{aligned}
& \frac{\partial}{\partial \eta}\left[\mathscr{M}_{\alpha / 2}\left(\frac{x}{\lambda(\eta-\tau)^{\alpha / 2}}\right) \frac{\alpha x}{2 \lambda(\eta-\tau)^{\alpha / 2+1}}\right] \frac{g(\tau)}{(t-\eta)^{\alpha}} \\
& \quad \in L^{1}(\Omega)
\end{aligned}
$$

where $\Omega=\left\{(\eta, \tau) \in \mathbb{R}^{2}: \eta \in(0, t), 0 \leq \tau \leq \eta\right\}$. Or equivalently,

$$
\begin{aligned}
& \frac{\partial}{\partial \eta}\left[\mathscr{M}_{\alpha / 2}\left(\frac{x}{\lambda(\eta-\tau)^{\alpha / 2}}\right)\right]\left(\frac{\alpha x}{2 \lambda(\eta-\tau)^{\alpha / 2+1}}\right) \\
& \cdot \frac{g(\tau)}{(t-\eta)^{\alpha}}-\mathscr{M}_{\alpha / 2}\left(\frac{x}{\lambda(\eta-\tau)^{\alpha / 2}}\right) \frac{(\alpha / 2+1) \alpha x}{2 \lambda(\eta-\tau)^{\alpha / 2+2}} \\
& \cdot \frac{g(\tau)}{(t-\eta)^{\alpha}}=I+I I \in L^{1}(\Omega) .
\end{aligned}
$$

Applying a similar reasoning like in the previous item, using Corollary 6, inequality (64), Corollary 9, and Tonelli's theorem (see [21], page 55), the following assertions are true:

$$
\int_{0}^{\eta}|I+I I| d \tau<\infty \quad \forall \eta \in(0, t) .
$$

Taking $\delta$ small according to Corollary 6 ,

$$
\begin{aligned}
\int_{0}^{t} \int_{0}^{\eta}|I+I I| d \tau d \eta= & \int_{0}^{t} \int_{0}^{\eta-\delta}|I+I I| d \tau d \eta \\
& +\int_{0}^{t} \int_{\eta-\delta}^{\eta}|I+I I| d \tau d \eta .
\end{aligned}
$$

Now, note that

$$
\begin{aligned}
\frac{\partial}{\partial \eta} & {\left[\mathscr{M}_{\alpha / 2}\left(\frac{x}{\lambda(\eta-\tau)^{\alpha / 2}}\right)\right] } \\
& =-\frac{\partial}{\partial \tau}\left[\mathscr{M}_{\alpha / 2}\left(\frac{x}{\lambda(\eta-\tau)^{\alpha / 2}}\right)\right]
\end{aligned}
$$

and that

$$
-\frac{\partial}{\partial \tau} \mathscr{M}_{\alpha / 2}\left(\frac{x}{\lambda(\eta-\tau)^{\alpha / 2}}\right)>0
$$

(it is a consequence of Lemma 4.2 from [17]).
Let $M$ be defined in (60) and let $C$ be any constant depending on $\delta, \alpha, x$, or $n$. Then

$$
\begin{aligned}
& \int_{0}^{t} \int_{0}^{\eta-\delta}|I| d \tau d \eta \leq \int_{0}^{t} \frac{M C}{(t-\eta)^{\alpha} \delta^{\alpha / 2+1}} \\
& \cdot \int_{0}^{\eta-\delta}-\frac{\partial}{\partial \tau} \mathscr{M}_{\alpha / 2}\left(\frac{x}{\lambda(\eta-\tau)^{\alpha / 2}}\right) d \tau d \eta \\
& \quad=\int_{0}^{t} \frac{M C}{(t-\eta)^{\alpha} \delta^{\alpha / 2+1}} \\
& \cdot\left[\mathscr{M}_{\alpha / 2}\left(\frac{x}{\lambda \eta^{\alpha / 2}}\right)-\mathscr{M}_{\alpha / 2}\left(\frac{x}{\lambda \delta^{\alpha / 2}}\right)\right] d \eta \\
& \quad \leq \frac{2 M C}{\Gamma(1-\alpha / 2) \delta^{\alpha / 2+1}} \int_{0}^{t} \frac{1}{(t-\eta)^{\alpha}} d \eta<\infty
\end{aligned}
$$

due to Lemma $4.2[17]$ and that $\alpha \in(0,1)$. Then,

$$
\int_{0}^{t} \int_{0}^{\eta-\delta}|I| d \tau d \eta<\infty
$$

On the other hand,

$$
\begin{aligned}
& \int_{0}^{t} \int_{0}^{\eta-\delta}|I I| d \tau d \eta=\int_{0}^{t} \int_{0}^{\eta-\delta} \mid \mathscr{M}_{\alpha / 2}\left(\frac{x}{\lambda(\eta-\tau)^{\alpha / 2}}\right) \\
& \cdot \frac{(\alpha / 2+1) \alpha x}{2 \lambda(t-\tau)^{\alpha / 2+2}} \frac{g(\tau)}{(t-\eta)^{\alpha}} \mid d \tau d \eta \\
& =\int_{0}^{t} \frac{1}{(t-\eta)^{\alpha}} \int_{0}^{\eta-\delta} \mid \mathscr{M}_{\alpha / 2}\left(\frac{x}{\lambda(\eta-\tau)^{\alpha / 2}}\right) \\
& \cdot \frac{(\alpha / 2+1) \alpha x}{2 \lambda(t-\tau)^{\alpha / 2+2}} g(\tau) \mid d \tau d \eta .
\end{aligned}
$$

We proved in the previous item that

$$
\begin{aligned}
& \int_{0}^{\eta}\left|\mathscr{M}_{\alpha / 2}\left(\frac{x}{\lambda(\eta-\tau)^{\alpha / 2}}\right) \frac{(\alpha / 2+1) \alpha x}{2 \lambda(\eta-\tau)^{\alpha / 2+2}} g(\tau)\right| d \tau \\
& \quad<\infty .
\end{aligned}
$$

Recalling that $\alpha \in(0,1)$, it yields that

$$
\int_{0}^{t} \int_{0}^{\eta-\delta}|I I| d \tau d \eta<\infty
$$

Then

$$
\int_{0}^{t} \int_{0}^{\eta-\delta}|I+I I| d \tau d \eta<\infty
$$

Proceeding like in item (ii), when checking Hypothesis (46), it can be proved that

$$
\int_{0}^{t} \int_{\eta-\delta}^{\eta}|I I| d \tau d \eta<\infty
$$


Finally, (79) and (80) yield

$$
\int_{0}^{t} \int_{0}^{\eta}|I+I I| d \tau d \eta<\infty
$$

From (75) and (81), Tonelli's theorem holds and

$$
\begin{gathered}
\frac{\partial}{\partial \eta}\left[\mathscr{M}_{\alpha / 2}\left(\frac{x}{\lambda(\eta-\tau)^{\alpha / 2}}\right) \frac{x}{\lambda(\eta-\tau)^{\alpha / 2+1}} \frac{\alpha}{2}\right] \\
\cdot \frac{g(\tau)}{(t-\eta)^{\alpha}} \in L^{1}(\Omega) .
\end{gathered}
$$

(iv) Hypothesis (48). Let us prove that

$$
\lim _{\tau \supset \eta} \mathscr{M}_{\alpha / 2}\left(\frac{x}{\lambda(\eta-\tau)^{\alpha / 2}}\right) \frac{\alpha x}{2 \lambda(\eta-\tau)^{\alpha / 2+1}} g(\tau)=0 .
$$

Note that, due to (59) and (60),

$$
\begin{aligned}
& \lim _{\tau \nearrow \eta} \mathscr{M}_{\alpha / 2}\left(\frac{x}{\lambda(\eta-\tau)^{\alpha / 2}}\right) \frac{\alpha x}{2 \lambda(\eta-\tau)^{\alpha / 2+1}} g(\tau) \\
& \quad=\lim _{s \rightarrow+0} \mathscr{M}_{\alpha / 2}\left(\frac{x}{\lambda s^{\alpha / 2}}\right) \frac{\alpha x}{2 \lambda s^{\alpha / 2+1}} g(\eta-s) \\
& \quad=\lim _{y \rightarrow \infty} \mathscr{M}_{\alpha / 2}\left(\frac{x}{\lambda} y^{\alpha / 2}\right) \frac{\alpha x y^{\alpha / 2+1}}{2 \lambda} g\left(\eta-\frac{1}{y^{\alpha / 2+1}}\right) \\
& =0 .
\end{aligned}
$$

Finally, we can apply Lemma 12 to kernel (44). Then,

$$
\begin{aligned}
& { }_{0} D^{\alpha} c_{2}(x, t)={ }_{0} D^{\alpha}\left(\int_{0}^{t} \mathscr{M}_{\alpha / 2}\left(\frac{x}{\lambda(t-\tau)^{\alpha / 2}}\right)\right. \\
& \left.\cdot \frac{\alpha x}{2 \lambda(t-\tau)^{\alpha / 2+1}} g(\tau) d \tau\right) \\
& =\int_{0}^{t}{ }_{0} D^{\alpha}\left(\mathscr{M}_{\alpha / 2}\left(\frac{x}{\lambda(t-\tau)^{\alpha / 2}}\right) \frac{\alpha x}{2 \lambda(t-\tau)^{\alpha / 2+1}}\right. \\
& \cdot g(\tau)) d \tau+I_{t}^{1-\alpha}(0)=\int_{0}^{t}{ }_{0} D^{\alpha}\left(-\frac{\partial}{\partial t}\right. \\
& \left.\cdot \mathscr{W}\left(\frac{-x}{\lambda(t-\tau)^{\alpha / 2}},-\frac{\alpha}{2}, 1\right)\right) g(\tau) d \tau \\
& =\int_{0}^{t}{ }_{0} D^{\alpha+1}\left(-\mathscr{W}\left(\frac{-x}{\lambda(t-\tau)^{\alpha / 2}},-\frac{\alpha}{2}, 1\right)\right) \\
& \cdot g(\tau) d \tau .
\end{aligned}
$$

From Corollary 5, $(\partial / \partial \tau) \mathscr{W}\left(-x / \lambda 0^{+},-\alpha / 2,1\right)=0$. Then Caputo derivative commutes (see equation (2.145) of [9]) and

$$
\begin{aligned}
{ }_{0} D^{\alpha+1}\left(-\mathscr{W}\left(\frac{-x}{\lambda(t-\tau)^{\alpha / 2}},-\frac{\alpha}{2}, 1\right)\right) \\
\quad={ }_{0} D^{1+\alpha}\left(-\mathscr{W}\left(\frac{-x}{\lambda(t-\tau)^{\alpha / 2}},-\frac{\alpha}{2}, 1\right)\right) \\
=-\frac{\partial}{\partial t}\left({ }_{0} D^{\alpha} \mathscr{W}\left(\frac{-x}{\lambda(t-\tau)^{\alpha / 2}},-\frac{\alpha}{2}, 1\right)\right) .
\end{aligned}
$$

Now, recalling that $\mathscr{W}\left(-x / \lambda(t-\tau)^{\alpha / 2},-\alpha / 2,1\right)$ is a solution of problem (40), it results in the following:

$$
\begin{aligned}
& -\frac{\partial}{\partial t}\left({ }_{0} D^{\alpha} \mathscr{W}\left(\frac{-x}{\lambda(t-\tau)^{\alpha / 2}},-\frac{\alpha}{2}, 1\right)\right) \\
& =-\frac{\partial}{\partial t} \frac{\partial}{\partial x^{2}} \mathscr{W}\left(\frac{-x}{\lambda(t-\tau)^{\alpha / 2}},-\frac{\alpha}{2}, 1\right) \\
& =\frac{\partial}{\partial x^{2}}\left(-\frac{\partial}{\partial t} \mathscr{W}\left(\frac{-x}{\lambda(t-\tau)^{\alpha / 2}},-\frac{\alpha}{2}, 1\right)\right) \\
& =\frac{\partial}{\partial x^{2}} \mathscr{M}_{\alpha / 2}\left(\frac{x}{\lambda(t-\tau)^{\alpha / 2}}\right) \frac{x}{\lambda(t-\tau)^{\alpha / 2+1}} \frac{\alpha}{2} .
\end{aligned}
$$

Replacing (87) in (85), we have

$$
\begin{aligned}
& { }_{0} D^{\alpha} c_{2}(x, t)=\int_{0}^{t} \frac{\partial}{\partial x^{2}} \mathscr{M}_{\alpha / 2}\left(\frac{x}{\lambda(t-\tau)^{\alpha / 2}}\right) \\
& \cdot \frac{x}{\lambda(t-\tau)^{\alpha / 2+1}} \frac{\alpha}{2} g(\tau) d \tau=\frac{\partial}{\partial x^{2}} \\
& \quad \cdot \int_{0}^{t} \mathscr{M}_{\alpha / 2}\left(\frac{x}{\lambda(t-\tau)^{\alpha / 2}}\right) \frac{x}{\lambda(t-\tau)^{\alpha / 2+1}} \frac{\alpha}{2} g(\tau) d \tau \\
& =\frac{\partial}{\partial x^{2}} c_{2}(x, t) .
\end{aligned}
$$

Therefore $c_{2}(x, t)$ verifies the fractional diffusion equation.

Proposition 13. The following limits hold:

(1) $\lim _{x \searrow 0} \int_{0}^{t} \mathscr{M}_{\alpha / 2}\left(x / \lambda(t-\tau)^{\alpha / 2}\right)\left(\alpha x / 2 \lambda(t-\tau)^{\alpha / 2+1}\right) d \tau=$ 1.

(2) $\lim _{t \searrow 0} \int_{0}^{t} \mathscr{M}_{\alpha / 2}\left(x / \lambda(t-\tau)^{\alpha / 2}\right)\left(\alpha x / 2 \lambda(t-\tau)^{\alpha / 2+1}\right) d \tau=$ 0 . 
Proof. (1) Taking $n=0$ in (59), it yields $\int_{0}^{\infty} \mathscr{M}_{\alpha / 2}(u) d u=1$. Making the substitution (63) and applying Corollary 9, we have

$$
\begin{aligned}
& \left|\int_{0}^{t} \mathscr{M}_{\alpha / 2}\left(\frac{x}{\lambda(t-\tau)^{\alpha / 2}}\right) \frac{\alpha x}{2 \lambda(t-\tau)^{\alpha / 2+1}} d \tau-1\right| \\
& \quad=\left|\int_{x / \lambda t^{\alpha / 2}}^{\infty} \mathscr{M}_{\alpha / 2}(u) d u-\int_{0}^{\infty} \mathscr{M}_{\alpha / 2}(u) d u\right| \\
& \quad \leq \int_{0}^{x / \lambda t^{\alpha / 2}} \mathscr{M}_{\alpha / 2}(u) d u \leq \frac{1}{\Gamma(1-\alpha / 2)} \frac{x}{\lambda t^{\alpha / 2}} \longrightarrow 0
\end{aligned}
$$

if $x \searrow 0$,

(2) It is a consequence of applying substitution (63).

Let us check now the border conditions.

(i) Consider $c_{2}(x, 0)=\lim _{t \backslash 0} c_{2}(x, t)$.

From Proposition 13 and (60),

$$
\begin{aligned}
0 & \leq \lim _{t \backslash 0} \mid \int_{0}^{t} \mathscr{M}_{\alpha / 2}\left(\frac{x}{\lambda(t-\tau)^{\alpha / 2}}\right) \\
& \cdot \frac{\alpha x}{2 \lambda(t-\tau)^{\alpha / 2+1}} g(\tau) d \tau \mid \\
& \leq \lim _{t \backslash 0} \int_{0}^{t} \mathscr{M}_{\alpha / 2}\left(\frac{x}{\lambda(t-\tau)^{\alpha / 2}}\right) \\
& \cdot \frac{\alpha x}{2 \lambda(t-\tau)^{\alpha / 2+1}} \frac{\alpha}{2}|g(\tau)| d \tau \\
& \leq \lim _{t \backslash 0} M \int_{0}^{t} \mathscr{M}_{\alpha / 2}\left(\frac{x}{\lambda(t-\tau)^{\alpha / 2}}\right) \\
& \cdot \frac{\alpha x}{2 \lambda(t-\tau)^{\alpha / 2+1}} \frac{\alpha}{2} d \tau=0 \\
\therefore & c_{2}(x, 0)=0 .
\end{aligned}
$$

(ii) Consider $c_{2}(0, t)=\lim _{x \backslash 0} \mathcal{c}_{2}(x, t)$.

Note that

$$
\begin{aligned}
& \int_{0}^{t} \mathscr{M}_{\alpha / 2}\left(\frac{\alpha x}{2 \lambda(t-\tau)^{\alpha / 2}}\right) \frac{x}{\lambda(t-\tau)^{\alpha / 2+1}} \frac{\alpha}{2} g(\tau) d \tau \\
& \quad=\int_{0}^{t} \mathscr{M}_{\alpha / 2}\left(\frac{x}{\lambda(t-\tau)^{\alpha / 2}}\right) \\
& \cdot \frac{\alpha x}{2 \lambda(t-\tau)^{\alpha / 2+1} \frac{\alpha}{2}[g(\tau)-g(t)+g(t)] d \tau} \\
& \quad=\int_{0}^{t} \mathscr{M}_{\alpha / 2}\left(\frac{x}{\lambda(t-\tau)^{\alpha / 2}}\right) \\
& \cdot \frac{\alpha x}{2 \lambda(t-\tau)^{\alpha / 2+1}}[g(\tau)-g(t)] d \tau \\
& +\int_{0}^{t} \mathscr{M}_{\alpha / 2}\left(\frac{x}{\lambda(t-\tau)^{\alpha / 2}}\right) \\
& \cdot \frac{\alpha x}{2 \lambda(t-\tau)^{\alpha / 2+1}} g(t) d \tau .
\end{aligned}
$$

Applying Proposition 13 to the second member of the sum,

$$
\begin{aligned}
& \lim _{x \searrow 0} \int_{0}^{t} \mathscr{M}_{\alpha / 2}\left(\frac{x}{\lambda(t-\tau)^{\alpha / 2}}\right) \frac{\alpha x}{2 \lambda(t-\tau)^{\alpha / 2+1}} g(t) d \tau \\
& \quad=g(t) \\
& \quad \cdot \lim _{x \unlhd 0} \int_{0}^{t} \mathscr{M}_{\alpha / 2}\left(\frac{x}{\lambda(t-\tau)^{\alpha / 2}}\right) \frac{\alpha x}{2 \lambda(t-\tau)^{\alpha / 2+1}} d \tau \\
& \quad=g(t) .
\end{aligned}
$$

Let

$$
\begin{aligned}
I= & \int_{0}^{t} \mathscr{M}_{\alpha / 2}\left(\frac{x}{\lambda(t-\tau)^{\alpha / 2}}\right) \\
& \cdot \frac{x}{\lambda(t-\tau)^{\alpha / 2+1}} \frac{\alpha}{2}[g(\tau)-g(t)] d \tau .
\end{aligned}
$$

The next goal is to prove that $\lim _{x \backslash 0} I=0$.

Let $\delta>0$ be determined. Consider

$$
\begin{aligned}
I= & \int_{0}^{t-\delta} \mathscr{M}_{\alpha / 2}\left(\frac{x}{\lambda(t-\tau)^{\alpha / 2}}\right) \\
& \cdot \frac{\alpha x}{2 \lambda(t-\tau)^{\alpha / 2+1}}[g(\tau)-g(t)] d \tau \\
& +\int_{t-\delta}^{t} \mathscr{M}_{\alpha / 2}\left(\frac{x}{\lambda(t-\tau)^{\alpha / 2}}\right) \\
& \cdot \frac{\alpha x}{2 \lambda(t-\tau)^{\alpha / 2+1}} \frac{\alpha}{2}[g(\tau)-g(t)] d \tau=I_{1}+I_{2} .
\end{aligned}
$$

Applying Corollary 9,

$$
\begin{aligned}
& \left|I_{1}\right|=\mid \int_{0}^{t-\delta} \mathscr{M}_{\alpha / 2}\left(\frac{x}{\lambda(t-\tau)^{\alpha / 2}}\right) \\
& \cdot \frac{\alpha x}{2 \lambda(t-\tau)^{\alpha / 2+1}}[g(\tau)-g(t)] d \tau \mid \\
& \quad \leq \int_{0}^{t-\delta} \frac{1}{\Gamma(1-\alpha / 2)} \frac{\alpha x}{2 \lambda(t-\tau)^{\alpha / 2+1}} \frac{\alpha}{2} \mid g(\tau) \\
& -g(t)\left|d \tau \leq \frac{1}{\Gamma(1-\alpha / 2)} \frac{\alpha x}{2 \lambda \delta^{\alpha / 2+1}} \int_{0}^{t-\delta}\right| g(\tau) \\
& -g(t) \mid d \tau \leq\left(\frac{1}{\Gamma(1-\alpha / 2)} \frac{\alpha}{2 \lambda \delta^{\alpha / 2+1}} \quad \text { if } x<\frac{\epsilon}{2 C_{t, \delta, \alpha}} .\right.
\end{aligned}
$$


Now, $g$ is continuous in $t$. Then, for $\epsilon>0$ given, there exists $\delta>0$ such that $|g(\tau)-g(t)|<\epsilon / 2$ if $|t-\tau|<\delta$. Using this fact and making the substitution (63),

$$
\begin{aligned}
\left|I_{2}\right| & \leq \frac{\epsilon}{2} \int_{t-\delta}^{t} \mathscr{M}_{\alpha / 2}\left(\frac{x}{\lambda(t-\tau)^{\alpha / 2}}\right) \frac{x}{\lambda(t-\tau)^{\alpha / 2+1}} \frac{\alpha}{2} d \tau \\
& =\frac{\epsilon}{2} \int_{x / \lambda \delta^{\alpha / 2}}^{\infty} \mathscr{M}_{\alpha / 2}(u) d u<\frac{\epsilon}{2} .
\end{aligned}
$$

From (95) and (96), it results that $|I|<\epsilon$, for every $\epsilon>0$. Then,

$$
\begin{aligned}
\lim _{x \searrow 0} \int_{0}^{t} \mathscr{M}_{\alpha / 2}\left(\frac{x}{\lambda(t-\tau)^{\alpha / 2}}\right) \\
\cdot \frac{x}{\lambda(t-\tau)^{\alpha / 2+1}} \frac{\alpha}{2}[g(\tau)-g(t)] d \tau=0,
\end{aligned}
$$

and we can assure that

$$
c_{2}(0, t)=g(t) .
$$

Theorem 14. Let $f$ be a continuous bounded function in $\mathbb{R}_{0}^{+}$ and let $g$ be a continuous function in $[0, T)$. Then

$$
\begin{aligned}
& c(x, t)=\int_{0}^{\infty} \frac{1}{2 \lambda t^{\alpha / 2}} \\
& \cdot\left[\mathscr{M}_{\alpha / 2}\left(\frac{|x-\xi|}{\lambda t^{\alpha / 2}}\right)-\mathscr{M}_{\alpha / 2}\left(\frac{|x+\xi|}{\lambda t^{\alpha / 2}}\right)\right] f(\xi) d \xi \\
& +\int_{0}^{t} \mathscr{M}_{\alpha / 2}\left(\frac{x}{\lambda(t-\tau)^{\alpha / 2}}\right) \frac{\alpha x}{2 \lambda(t-\tau)^{\alpha / 2+1}} g(\tau) d \tau
\end{aligned}
$$

is a solution to problem

$$
\begin{aligned}
D^{\alpha} c(x, t) & =\lambda^{2} \frac{\partial^{2} c}{\partial x^{2}}(x, t) \\
0<x<\infty, 0<t<T, 0<\alpha<1, & \\
c(x, 0) & =f(x) \quad 0<x<\infty, \\
c(0, t) & =g(t) \quad 0<t<T .
\end{aligned}
$$

Theorem 15. The limit when $\alpha \nearrow 1$ of the solution to problem

$$
\begin{aligned}
D^{\alpha} c_{2}(x, t) & =\lambda^{2} \frac{\partial^{2} c_{2}}{\partial x^{2}}(x, t) \\
& 0<x<\infty, 0<t<T, 0<\alpha<1, \\
c_{2}(x, 0) & =0 \quad 0<x<\infty, \\
c_{2}(0, t) & =g(t) \quad 0<t<T,
\end{aligned}
$$

is the classical solution to the analogous problem when $\alpha=1$ and we recover the heat equation

$$
\begin{aligned}
\frac{\partial}{\partial t} w(x, t) & =\lambda^{2} \frac{\partial^{2} c}{\partial x^{2}}(x, t) \quad 0<x<\infty, 0<t<T \\
w(x, 0) & =0 \quad 0<x<\infty \\
w(0, t) & =g(t) \quad 0<t<T .
\end{aligned}
$$

Proof. Let

$$
w(x, t)=\int_{0}^{t} \frac{e^{-x^{2} / 4 \lambda^{2}(t-\tau)}}{2 \sqrt{\pi}} \frac{x}{\lambda(t-\tau)^{3 / 2}} g(\tau) d \tau
$$

be the solution of problem (102) (see [1]) and let $c_{2}^{\alpha}$ be the solution of problem (101) given by Theorem 14,

$$
=\int_{0}^{t} \mathscr{M}_{\alpha / 2}\left(\frac{x}{\lambda(t-\tau)^{\alpha / 2}}\right) \frac{x}{\lambda(t-\tau)^{\alpha / 2+1}} \frac{\alpha}{2} g(\tau) d \tau .
$$

Applying Lebesgue Convergence Theorem and Lemma 11,

$$
\begin{aligned}
\lim _{\alpha \supset 1} c_{2}^{\alpha}(x, t)=\lim _{\alpha \supset 1}\left\{\int_{0}^{t} \mathscr{M}_{\alpha / 2}\left(\frac{x}{\lambda(t-\tau)^{\alpha / 2}}\right)\right. \\
\left.\cdot \frac{\alpha x}{2 \lambda(t-\tau)^{\alpha / 2+1}} g(\tau) d \tau\right\} \\
=\int_{0}^{t} \lim _{\alpha \supset 1} M_{\alpha / 2}\left(\frac{x}{\lambda(t-\tau)^{\alpha / 2}}\right) \frac{\alpha x}{2 \lambda(t-\tau)^{\alpha / 2+1}} \\
\cdot g(\tau) d \tau=\int_{0}^{t} \frac{e^{-x^{2} / 4(t-\tau)}}{2 \sqrt{\pi}} \frac{x}{\lambda(t-\tau)^{3 / 2}} g(\tau) d \tau \\
=w(x, t) .
\end{aligned}
$$

\section{Solving the Initial-Boundary-Value Problem for the Time-Fractional Diffusion Equation on the Real Positive Semiaxis with Null Flux-Boundary Condition}

In this section, we consider the problem

$$
\begin{aligned}
&{ }_{0} D^{\alpha} c_{3}(x, t)=\lambda^{2} \frac{\partial^{2} c_{3}}{\partial x^{2}}(x, t) \quad 0<x<\infty, 0<t<T, 0<\alpha<1, \\
& \frac{\partial c_{3}}{\partial x}(0, t)=0 \quad 0<t<T, \\
& c_{3}(x, 0)=f(x) \quad 0<x<\infty .
\end{aligned}
$$

Let us therefore consider the following auxiliary problem:

$$
\begin{aligned}
{ }_{0} D^{\alpha} z(x, t)= & \lambda^{2} \frac{\partial^{2} c}{\partial x^{2}}(x, t) \\
& \quad-\infty<x<\infty, 0<t<T, 0<\alpha<1 \\
z(x, 0)= & \tilde{f}(x)-\infty<x<\infty,
\end{aligned}
$$

where $\tilde{f}$ is an even extension of $f$.

This problem was solved in [22] and its solution is given by the following function:

$$
\begin{aligned}
c_{3}(x, t)=\int_{-\infty}^{\infty} \frac{t^{-\alpha / 2}}{2 \lambda} \mathscr{M}_{\alpha / 2}\left(\frac{|x-\xi|}{\lambda t^{\alpha / 2}}\right) \tilde{f}(\xi) d \xi \\
=\frac{1}{2 \lambda t^{\alpha / 2}} \\
\quad \cdot \int_{0}^{\infty}\left[\mathscr{M}_{\alpha / 2}\left(\frac{x+\xi}{\lambda t^{\alpha / 2}}\right)+\mathscr{M}_{\alpha / 2}\left(\frac{|x-\xi|}{\lambda t^{\alpha / 2}}\right)\right] \\
\cdot f(\xi) d \xi .
\end{aligned}
$$


The next goal is to prove that

$$
\begin{aligned}
& \lim _{x \searrow 0} \frac{\partial}{\partial x} c_{3}(x, t)=0 . \\
& \frac{\partial c_{3}}{\partial x}(0, t)=\lim _{x \searrow 0} \frac{1}{2 \lambda t^{\alpha / 2}} \frac{\partial}{\partial x} \int_{0}^{\infty}\left[\mathscr{M}_{\alpha / 2}\left(\frac{x+\xi}{\lambda t^{\alpha / 2}}\right)\right. \\
& \left.\quad+\mathscr{M}_{\alpha / 2}\left(\frac{|x-\xi|}{\lambda t^{\alpha / 2}}\right)\right] f(\xi) d \xi=\lim _{x \searrow 0} \frac{1}{2 \lambda t^{\alpha / 2}} \frac{\partial}{\partial x} \\
& \quad \cdot\left\{\int_{0}^{x}\left[\mathscr{M}_{\alpha / 2}\left(\frac{x+\xi}{\lambda t^{\alpha / 2}}\right)+\mathscr{M}_{\alpha / 2}\left(\frac{x-\xi}{\lambda t^{\alpha / 2}}\right)\right]\right. \\
& \quad \cdot f(\xi) d \xi \\
& \quad+\int_{x}^{\infty}\left[\mathscr{M}_{\alpha / 2}\left(\frac{x+\xi}{\lambda t^{\alpha / 2}}\right)+\mathscr{M}_{\alpha / 2}\left(\frac{\xi-x}{\lambda t^{\alpha / 2}}\right)\right] \\
& \quad \cdot f(\xi) d \xi\} .
\end{aligned}
$$

Due to the continuity of the Mainardi function, Corollary 6, and $f \in \mathscr{C}\left(\mathbb{R}^{+}\right) \cap L^{\infty}\left(\mathbb{R}^{+}\right)$, the next equalities are true:

$$
\begin{aligned}
& \frac{\partial}{\partial x} \int_{0}^{x}\left[M_{\alpha / 2}\left(\frac{x+\xi}{\lambda t^{\alpha / 2}}\right)+\mathscr{M}_{\alpha / 2}\left(\frac{x-\xi}{\lambda t^{\alpha / 2}}\right)\right] f(\xi) d \xi \\
& \quad=\int_{0}^{x} \frac{\partial}{\partial x}\left[\mathscr{M}_{\alpha / 2}\left(\frac{x+\xi}{\lambda t^{\alpha / 2}}\right)+\mathscr{M}_{\alpha / 2}\left(\frac{x-\xi}{\lambda t^{\alpha / 2}}\right)\right] \\
& \cdot f(\xi) d \xi \\
& \quad+\lim _{\xi>x}\left[\mathscr{M}_{\alpha / 2}\left(\frac{x+\xi}{\lambda t^{\alpha / 2}}\right)+\mathscr{M}_{\alpha / 2}\left(\frac{x-\xi}{\lambda t^{\alpha / 2}}\right)\right] f(\xi), \\
& \frac{\partial}{\partial x} \int_{x}^{\infty}\left[\mathscr{M}_{\alpha / 2}\left(\frac{x+\xi}{\lambda t^{\alpha / 2}}\right)+\mathscr{M}_{\alpha / 2}\left(\frac{\xi-x}{\lambda t^{\alpha / 2}}\right)\right] f(\xi) d \xi \\
& \quad=-\int_{\infty}^{x} \frac{\partial}{\partial x}\left[\mathscr{M}_{\alpha / 2}\left(\frac{x+\xi}{\lambda t^{\alpha / 2}}\right)+\mathscr{M}_{\alpha / 2}\left(\frac{\xi-x}{\lambda t^{\alpha / 2}}\right)\right] \\
& \quad \cdot f(\xi) d \xi \\
& \quad-\lim _{\xi \searrow x}\left[\mathscr{M}_{\alpha / 2}\left(\frac{x+\xi}{\lambda t^{\alpha / 2}}\right)+\mathscr{M}_{\alpha / 2}\left(\frac{\xi-x}{\lambda t^{\alpha / 2}}\right)\right] f(\xi) .
\end{aligned}
$$

Note that

$$
\begin{aligned}
& \int_{0}^{x} \frac{\partial}{\partial x}\left[\mathscr{M}_{\alpha / 2}\left(\frac{x+\xi}{\lambda t^{\alpha / 2}}\right)+\mathscr{M}_{\alpha / 2}\left(\frac{x-\xi}{\lambda t^{\alpha / 2}}\right)\right] f(\xi) d \xi \\
& \quad=-\frac{1}{\lambda t^{\alpha / 2}} \int_{0}^{x}\left[\mathscr{W}\left(-\frac{x+\xi}{\lambda t^{\alpha / 2}},-\frac{\alpha}{2}, 1-\alpha\right)\right. \\
& \left.\quad+\mathscr{W}\left(-\frac{x-\xi}{\lambda t^{\alpha / 2}},-\frac{\alpha}{2}, 1-\alpha\right)\right] f(\xi) d \xi .
\end{aligned}
$$

Applying Mean Value Theorem, it yields that

$$
\begin{aligned}
& \mid \int_{0}^{x}\left[\mathscr{W}\left(-\frac{x+\xi}{\lambda t^{\alpha / 2}},-\frac{\alpha}{2}, 1-\alpha\right)\right. \\
& \left.\quad+\mathscr{W}\left(-\frac{x-\xi}{\lambda t^{\alpha / 2}},-\frac{\alpha}{2}, 1-\alpha\right)\right] f(\xi) d \xi \mid
\end{aligned}
$$

$$
\begin{aligned}
& =||\left[\mathscr{W}\left(-\frac{x+\theta}{\lambda t^{\alpha / 2}},-\frac{\alpha}{2}, 1-\alpha\right)+\mathscr{W}\left(-\frac{x-\theta}{\lambda t^{\alpha / 2}},\right.\right. \\
& \left.\left.-\frac{\alpha}{2}, 1-\alpha\right)\right] f(\theta) x \mid \quad \theta \in[0, x] .
\end{aligned}
$$

Then,

$$
\begin{gathered}
\lim _{x \searrow 0} \int_{0}^{x} \frac{\partial}{\partial x}\left[\mathscr{M}_{\alpha / 2}\left(\frac{x+\xi}{\lambda t^{\alpha / 2}}\right)+\mathscr{M}_{\alpha / 2}\left(\frac{x-\xi}{\lambda t^{\alpha / 2}}\right)\right] \\
\cdot f(\xi) d \xi=0 .
\end{gathered}
$$

On the other side,

$$
\begin{aligned}
\int_{x}^{\infty} & \frac{\partial}{\partial x}\left[\mathscr{M}_{\alpha / 2}\left(\frac{x+\xi}{\lambda t^{\alpha / 2}}\right)+\mathscr{M}_{\alpha / 2}\left(\frac{\xi-x}{\lambda t^{\alpha / 2}}\right)\right] f(\xi) d \xi \\
& =\int_{x}^{\infty}-\frac{1}{\lambda t^{\alpha / 2}}\left[\mathscr{W}\left(-\frac{x+\xi}{\lambda t^{\alpha / 2}},-\frac{\alpha}{2}, 1-\alpha\right)\right. \\
& \left.-\mathscr{W}\left(-\frac{\xi-x}{\lambda t^{\alpha / 2}},-\frac{\alpha}{2}, 1-\alpha\right)\right] f(\xi) d \xi \\
& =\int_{0}^{\infty}-\frac{1}{\lambda t^{\alpha / 2}}\left[\mathscr{W}\left(-\frac{x+\xi}{\lambda t^{\alpha / 2}},-\frac{\alpha}{2}, 1-\alpha\right)\right. \\
& \left.-\mathscr{W}\left(-\frac{\xi-x}{\lambda t^{\alpha / 2}},-\frac{\alpha}{2}, 1-\alpha\right)\right] f(\xi) d \xi \\
& -\int_{0}^{x}-\frac{1}{\lambda t^{\alpha / 2}}\left[\mathscr{W}\left(-\frac{x+\xi}{\lambda t^{\alpha / 2}},-\frac{\alpha}{2}, 1-\alpha\right)\right. \\
& \left.-\mathscr{W}\left(-\frac{\xi-x}{\lambda t^{\alpha / 2}},-\frac{\alpha}{2}, 1-\alpha\right)\right] f(\xi) d \xi .
\end{aligned}
$$

Applying Lebesgue Convergence Theorem to the first integral,

$$
\begin{aligned}
\lim _{x \searrow 0} \int_{0}^{\infty}-\frac{1}{\lambda t^{\alpha / 2}}\left[\mathscr{W}\left(-\frac{x+\xi}{\lambda t^{\alpha / 2}},-\frac{\alpha}{2}, 1-\alpha\right)\right. \\
\left.-\mathscr{W}\left(-\frac{\xi-x}{\lambda t^{\alpha / 2}},-\frac{\alpha}{2}, 1-\alpha\right)\right] f(\xi) d \xi \\
\quad=\int_{0}^{\infty} \lim _{x \searrow 0}-\frac{1}{\lambda t^{\alpha / 2}}\left[\mathscr{W}\left(-\frac{x+\xi}{\lambda t^{\alpha / 2}},-\frac{\alpha}{2}, 1-\alpha\right)\right. \\
\left.-\mathscr{W}\left(-\frac{\xi-x}{\lambda t^{\alpha / 2}},-\frac{\alpha}{2}, 1-\alpha\right)\right] f(\xi) d \xi \\
=\int_{0}^{\infty}-\frac{1}{\lambda t^{\alpha / 2}}\left[\mathscr{W}\left(-\frac{\xi}{\lambda t^{\alpha / 2}},-\frac{\alpha}{2}, 1-\alpha\right)\right. \\
\left.-\mathscr{W}\left(-\frac{\xi}{\lambda t^{\alpha / 2}},-\frac{\alpha}{2}, 1-\alpha\right)\right] f(\xi) d \xi=0 .
\end{aligned}
$$

For the second integral we apply Mean Value Theorem as before. Then,

$$
\begin{aligned}
& \lim _{x \searrow 0} \int_{x}^{\infty} \frac{\partial}{\partial x}\left[\mathscr{M}_{\alpha / 2}\left(\frac{x+\xi}{\lambda t^{\alpha / 2}}\right)+\mathscr{M}_{\alpha / 2}\left(\frac{\xi-x}{\lambda t^{\alpha / 2}}\right)\right] \\
& \cdot f(\xi) d \xi=0 .
\end{aligned}
$$


From (113) and (116),

$$
\frac{\partial c_{3}}{\partial x}(0, t)=0
$$

Theorem 16. Let $f$ be a continuous bounded function in $\mathbb{R}_{0}^{+}$; then

$$
\begin{aligned}
& c(x, t)=\frac{1}{2 \lambda t^{\alpha / 2}} \\
& \quad \cdot \int_{0}^{\infty}\left[\mathscr{M}_{\alpha / 2}\left(\frac{x+\xi}{\lambda t^{\alpha / 2}}\right)+\mathscr{M}_{\alpha / 2}\left(\frac{|x-\xi|}{\lambda t^{\alpha / 2}}\right)\right] \\
& \quad \cdot f(\xi) d \xi
\end{aligned}
$$

is a solution to problem

$$
\begin{aligned}
D^{\alpha} c(x, t) & =\lambda^{2} \frac{\partial^{2} c}{\partial x^{2}}(x, t), \\
& 0<x<\infty, 0<t<T, 0<\alpha<1, \\
\frac{\partial c}{\partial x}(0, t) & =0, \quad 0<t<T, \\
c(x, 0) & =f(x), \quad 0<x<\infty .
\end{aligned}
$$

\section{The Initial-Boundary-Value Problem for the Time-Fractional Diffusion Equation on the Real Positive Semiaxis with Flux-Boundary Condition}

As in the previous sections two auxiliary problems are considered:

$$
\begin{aligned}
&{ }_{0} D_{t}^{\alpha} c_{4}(x, t)= \lambda^{2} \frac{\partial^{2} c_{1}}{\partial x^{2}}(x, t) \\
& 0<x<\infty, 0<t<T, 0<\alpha<1 \\
& c_{4}(x, 0)=f(x) \quad 0<x<\infty \\
& \frac{\partial}{\partial x} c_{4}(0, t)=0 \quad 0<t<T, \\
&{ }_{0} D_{t}^{\alpha} c_{5}(x, t)=\lambda^{2} \frac{\partial^{2} c_{2}}{\partial x^{2}}(x, t) \\
& \quad 0<x<\infty, 0<t<T, 0<\alpha<1 \\
& c_{5}(x, 0)=0 \quad 0<x<\infty \\
& \frac{\partial}{\partial x} c_{5}(0, t)=g(t) \quad 0<t<T .
\end{aligned}
$$

From Theorem 16,

$$
\begin{aligned}
& c_{4}(x, t)=\frac{1}{2 \lambda t^{\alpha / 2}} \\
& \cdot \int_{0}^{\infty}\left[M_{\alpha / 2}\left(\frac{x+\xi}{\lambda t^{\alpha / 2}}\right)+M_{\alpha / 2}\left(\frac{|x-\xi|}{\lambda t^{\alpha / 2}}\right)\right]
\end{aligned}
$$

$$
\cdot f(\xi) d \xi
$$

is a solution to (120).
In view of the results obtained in Section 3

$$
\begin{gathered}
c_{5}(x, t)=-\int_{x}^{\infty} \int_{0}^{t} \mathscr{M}_{\alpha / 2}\left(\frac{x}{\lambda(t-\tau)^{\alpha / 2}}\right) \\
\cdot \frac{x}{\lambda(t-\tau)^{\alpha / 2+1}} \frac{\alpha}{2} g(\tau) d \tau d \xi
\end{gathered}
$$

is proposed as a solution to problem (121).

Lemma 17. Let $c(x, t)$ be a solution of the time-fractional diffusion equation ${ }_{0} D_{t}^{\alpha} c(x, t)=\lambda^{2}\left(\partial^{2} c / \partial x^{2}\right)(x, t)$ such that

(i) For every $(x, t)$, the function $F(x, t)$

$$
=\int_{x}^{\infty} c(\xi, t) d \xi \text { is well defined, }
$$

(ii) $\lim _{x \rightarrow \infty} \frac{\partial c}{\partial x}(x, t)=0$,

(iii) $\left|\frac{\partial}{\partial \tau} c(\xi, \tau)\right| \leq k(\xi) \in L^{1}(x, \infty)$,

$$
\text { (iv) } \frac{(\partial / \partial \tau) c(\xi, \tau)}{(t-\tau)^{\alpha}} \in L^{1}((x, \infty) \times(0, t)) \text {. }
$$

Then $\int_{x}^{\infty} c(\xi, t) d \xi$ is a solution to the time fractional diffusion equation.

Proof. From (124), $F(x, t)=\int_{x}^{\infty} c(\xi, t) d \xi$ is well defined. The next equalities are valid due to (125), (126), and (127):

$$
\begin{aligned}
&{ }_{0} D_{t}^{\alpha} F(x, t)=\frac{1}{\Gamma(1-\alpha)} \int_{0}^{t} \frac{(\partial / \partial \tau) F(x, \tau)}{(t-\tau)^{\alpha}} d \tau \\
&=\frac{1}{\Gamma(1-\alpha)} \int_{0}^{t} \frac{1}{(t-\tau)^{\alpha}}\left(\frac{\partial}{\partial \tau} \int_{x}^{\infty} c(\xi, \tau) d \xi\right) d \tau \\
&= \frac{1}{\Gamma(1-\alpha)} \int_{0}^{t} \frac{1}{(t-\tau)^{\alpha}} \int_{x}^{\infty} \frac{\partial}{\partial \tau} c(\xi, \tau) d \xi d \tau \\
&=\int_{x}^{\infty} \frac{1}{\Gamma(1-\alpha)} \int_{0}^{t} \frac{(\partial / \partial \tau) c(\xi, \tau)}{(t-\tau)^{\alpha}} d \tau \\
&=\int_{x}^{\infty}{ }_{0}^{\infty} c(\xi, t) d \xi=\int_{x}^{\infty} \lambda^{2} \frac{\partial^{2} c}{\partial x^{2}}(\xi, t) d \xi \\
&=-\left.\lambda^{2} \frac{\partial c}{\partial x}(\xi, t)\right|_{\infty} ^{x} \\
&=-\lambda^{2} \frac{\partial c}{\partial x}(x, t)+\lambda^{2} \lim _{x \rightarrow \infty} \frac{\partial c}{\partial x}(x, t) \\
&=\frac{\partial^{2}}{\partial x^{2}}\left(\int_{x}^{\infty} c(\xi, t) d \xi\right)=\frac{\partial^{2}}{\partial x^{2}} F(x, t) .
\end{aligned}
$$

It can be proved that (123) is under the hypothesis of Lemma 17.

Regarding the border conditions: 
(i) Observing that

$$
\begin{aligned}
& \int_{0}^{t} \mathscr{M}_{\alpha / 2}\left(\frac{\xi}{\lambda(t-\tau)^{\alpha / 2}}\right) \frac{\xi}{\lambda(t-\tau)^{\alpha / 2+1}} \frac{\alpha}{2} g(\tau) d \tau \\
& \quad \leq M W\left(-\frac{\xi}{\lambda t^{\alpha / 2}},-\frac{\alpha}{2}, 1\right),
\end{aligned}
$$

Lebesgue Convergence Theorem can be applied and

$$
\begin{aligned}
& c_{5}(x, 0) \\
& =\lim _{t \searrow 0}-\int_{x}^{\infty} \int_{0}^{t} \mathscr{M}_{\alpha / 2}\left(\frac{\xi}{\lambda(t-\tau)^{\alpha / 2}}\right) \frac{\xi}{\lambda(t-\tau)^{\alpha / 2+1}} \frac{\alpha}{2} g(\tau) d \tau d \xi \\
& =\lim _{t \searrow 0}\left|\int_{x}^{\infty} \int_{0}^{t} \mathscr{M}_{\alpha / 2}\left(\frac{\xi}{\lambda(t-\tau)^{\alpha / 2}}\right) \frac{\xi}{\lambda(t-\tau)^{\alpha / 2+1}} \frac{\alpha}{2} g(\tau) d \tau d \xi\right| \\
& =\left|\int_{x}^{\infty} \lim _{t \backslash 0} \int_{0}^{t} \mathscr{M}_{\alpha / 2}\left(\frac{\xi}{\lambda(t-\tau)^{\alpha / 2}}\right) \frac{\xi}{\lambda(t-\tau)^{\alpha / 2+1}} \frac{\alpha}{2} g(\tau) d \tau d \xi\right| \\
& \leq \int_{x}^{\infty}\left|\lim _{t \backslash 0} M \mathscr{W}\left(-\frac{\xi}{\lambda t^{\alpha / 2}},-\frac{\alpha}{2}, 1\right)\right| d \xi=0 .
\end{aligned}
$$

(ii) From (98),

$$
\begin{gathered}
\frac{\partial}{\partial x} c_{5}(0, t)=\lim _{x \searrow 0} \frac{\partial}{\partial x}\left(-\int_{x}^{\infty} \int_{0}^{t} \mathscr{M}_{\alpha / 2}\left(\frac{\xi}{\lambda(t-\tau)^{\alpha / 2}}\right)\right. \\
\left.\cdot \frac{\xi}{\lambda(t-\tau)^{\alpha / 2+1}} \frac{\alpha}{2} g(\tau) d \tau d \xi\right) \\
=\lim _{x \searrow 0} \int_{0}^{t} \mathscr{M}_{\alpha / 2}\left(\frac{x}{\lambda(t-\tau)^{\alpha / 2}}\right) \\
\cdot \frac{x}{\lambda(t-\tau)^{\alpha / 2+1}} \frac{\alpha}{2} g(\tau) d \tau=g(t) .
\end{gathered}
$$

Theorem 18. Let $f$ be a continuous bounded function in $\mathbb{R}_{0}^{+}$ and let $g$ be a continuous function in $[0, T)$. Then

$$
\begin{gathered}
c(x, t)=\frac{1}{2 \lambda t^{\alpha / 2}} \int_{0}^{\infty}\left[\mathscr{M}_{\alpha / 2}\left(\frac{x+\xi}{\lambda t^{\alpha / 2}}\right)\right. \\
\left.+\mathscr{M}_{\alpha / 2}\left(\frac{|x-\xi|}{\lambda t^{\alpha / 2}}\right)\right] f(\xi) d \xi \\
-\int_{x}^{\infty} \int_{0}^{t} \mathscr{M}_{\alpha / 2}\left(\frac{x}{\lambda(t-\tau)^{\alpha / 2}}\right) \\
\cdot \frac{x}{\lambda(t-\tau)^{\alpha / 2+1}} \frac{\alpha}{2} g(\tau) d \tau d \xi
\end{gathered}
$$

is a solution to problem

$$
\begin{aligned}
D^{\alpha} c(x, t) & =\lambda^{2} \frac{\partial^{2} c}{\partial x^{2}}(x, t), \\
0<x<\infty, 0<t<T, 0<\alpha<1, & \\
c(x, 0) & =f(x), \quad 0<x<\infty, \\
\frac{\partial}{\partial x} c(0, t) & =g(t), \quad 0<t<T .
\end{aligned}
$$

Theorem 19. The limit when $\alpha \nearrow 1$ of the solution to problem

$$
\begin{aligned}
D^{\alpha} c_{5}(x, t) & =\lambda^{2} \frac{\partial^{2} c_{2}}{\partial x^{2}}(x, t) \\
0 & <x<\infty, 0<t<T, 0<\alpha<1, \\
\mathcal{c}_{5}(x, 0) & =0, \quad 0<x<\infty \\
\frac{\partial}{\partial x} c_{5}(0, t) & =g(t), \quad 0<t<T
\end{aligned}
$$

is the classical solution to the analogous problem when $\alpha=1$ and we recover the heat equation

$$
\begin{aligned}
\frac{\partial}{\partial t} w(x, t) & =\lambda^{2} \frac{\partial^{2} c}{\partial x^{2}}(x, t), \quad 0<x<\infty, \quad 0<t<T \\
w(x, 0) & =0, \quad 0<x<\infty \\
\frac{\partial}{\partial x} w(0, t) & =g(t), \quad 0<t<T .
\end{aligned}
$$

Proof. It can be seen in [1] that

$$
w(x, t)=-\int_{0}^{t} \frac{e^{-x^{2} / 4(t-\tau)}}{\sqrt{\pi(t-\tau)}} g(\tau) d \tau
$$

is a solution to problem (135).

Let $c_{5}^{\alpha}$ be a solution to problem (134) given by Theorem 18 ,

$$
\begin{gathered}
c_{5}^{\alpha}(x, t)=-\int_{x}^{\infty} \int_{0}^{t} \mathscr{M}_{\alpha / 2}\left(\frac{x}{\lambda(t-\tau)^{\alpha / 2}}\right) \\
\cdot \frac{x}{\lambda(t-\tau)^{\alpha / 2+1}} \frac{\alpha}{2} g(\tau) d \tau d \xi .
\end{gathered}
$$

Applying Lebesgue Convergence Theorem, Lemma 11, and Fubini's Theorem,

$$
\begin{aligned}
\lim _{\alpha>1} c_{5}^{\alpha}(x, t) & =\lim _{\alpha>1}\left\{-\int_{x}^{\infty} \int_{0}^{t} \mathscr{M}_{\alpha / 2}\left(\frac{x}{\lambda(t-\tau)^{\alpha / 2}}\right) \frac{x}{\lambda(t-\tau)^{\alpha / 2+1}} \frac{\alpha}{2} g(\tau) d \tau d \xi\right\} \\
& =-\int_{x}^{\infty} \int_{0}^{t} \lim _{\alpha>1} \mathscr{M}_{\alpha / 2}\left(\frac{x}{\lambda(t-\tau)^{\alpha / 2}}\right) \frac{x}{\lambda(t-\tau)^{\alpha / 2+1}} \frac{\alpha}{2} g(\tau) d \tau d \xi=-\int_{x}^{\infty} \int_{0}^{t} \frac{e^{-x^{2} / 4(t-\tau)}}{2 \sqrt{\pi}} \frac{x}{\lambda(t-\tau)^{3 / 2}} g(\tau) d \tau \\
& =-\int_{0}^{t} \int_{x}^{\infty} \frac{e^{-x^{2} / 4(t-\tau)}}{2 \sqrt{\pi}} \frac{x}{\lambda(t-\tau)^{3 / 2}} g(\tau) d \tau=-\int_{0}^{t} \frac{e^{-x^{2} / 4(t-\tau)}}{\sqrt{\pi(t-\tau)}} g(\tau) d \tau=w(x, t) .
\end{aligned}
$$




\section{Conclusions}

On the basis of the asymptotic behavior of some Wright functions and the existence of bounds for the Mainardi and the Wright function $W(-x, \alpha / 2,1)$ in $\mathbb{R}^{+}$, two different initialboundary-value problems (with Dirichlet and Neumann boundary conditions, resp.) for the time-fractional diffusion equation on the real positive semiaxis plane were solved. In each case, certain conditions must be verified for the data to obtain the solution and the convergence of this solution when $\alpha \nearrow 1$ was analyzed, recovering the classical solutions of the respective boundary-value problems corresponding to the heat equation on the real positive semiaxis.

It would be interesting to combine these obtained results for the two initial-boundary-value problems with numerical methods that approximate their solutions. Different authors have focused their work on fractional numerical methods, for example, [23], considering explicitly the time-fractional diffusion equation or also [24, 25] providing different approaches to these kind of problems. Taking into account that the explicit expressions of the solutions provided in this work are manageable and of low complexity, they might help to check if new numerical algorithms actually converge to the desired solution of the problem and at which rate.

\section{Conflict of Interests}

The authors declare that there is no conflict of interests regarding the publication of this paper.

\section{Acknowledgment}

This paper has been sponsored by the Project ING495 "Estudio de Diversos Problemas con Ecuaciones Diferenciales Fraccionarias" from Universidad Nacional de Rosario, Argentina.

\section{References}

[1] J. R. Cannon, The One-Dimensional Heat Equation, Cambridge University Press, Cambridge, UK, 1984.

[2] A. Friedman, Partial Differential Equations of Parabolic Type, Prentice-Hall, Englewood Cliffs, NJ, USA, 1964.

[3] D. V. Widder, The Heat Equation, Academic Press, New York, NY, USA, 1975.

[4] K. Diethelm, The Analysis of Fractional Differential Equations: An Application Oriented Exposition Using Differential Operators of Caputo Typ, Springer, 2010.

[5] Y. Fujita, "Integrodifferential equations which interpolates the heat equation and a wave equation," Osaka Journal of Mathematics, vol. 27, pp. 309-321, 1990.

[6] A. Kilbas, H. Srivastava, and J. Trujillo, Theory and Applications of Fractional Differential Equations, vol. 204 of North-Holland Mathematics Studies, Elsevier Science, Amsterdam, The Netherlands, 2006.

[7] Y. Luchko, "Some uniqueness and existence results for the initial-boundary-value problems for the generalized timefractional diffusion equation," Computers \& Mathematics with Applications, vol. 59, no. 5, pp. 1766-1772, 2010.
[8] F. Mainardi, Y. Luchko, and G. Pagnini, “The fundamental solution of the space-time fractional diffusion equation," Fractional Calculus \& Applied Analysis, vol. 4, no. 2, pp. 153-192, 2001.

[9] I. Podlubny, Fractional Differential Equations, vol. 198 of Mathematics in Science and Engineering, Academic Press, San Diego, Calif, USA, 1999.

[10] W. Wyss, “The fractional diffusion equation," Journal of Mathematical Physics, vol. 27, no. 11, pp. 2782-2785, 1986.

[11] F. Mainardi, Fractional Calculus and Waves in Linear Viscoelasticity, Imperial College Press, London, UK, 2010.

[12] S. D. Eidelman and A. N. Kochubei, "Cauchy problem for fractional diffusion equations," Journal of Differential Equations, vol. 199, no. 2, pp. 211-255, 2004.

[13] V. R. Voller, F. Falcini, and R. Garra, "Fractional Stefan problems exhibiting lumped and distributed latent-heat memory effects," Physical Review E, vol. 87, no. 4, Article ID 042401, 2013.

[14] Y. Povstenko, "Fractional heat conduction in a semi-infinite composite body", Communications in Applied and Industrial Mathematics, vol. 6, no. 1, p. e-482, 2014.

[15] F. Mainardi and M. Tomirotti, "On a special function arising in the time fractional diffusion-wave equation," in Transform Methods and Special Functions, Sofia 1994, P. Rusev, I. Dimovski, and V. Kiryakova, Eds., pp. 171-183, Science Culture Technology, Singapore, 1995.

[16] E. M. Wright, "The generalized Bessel function of order greater than one," Quarterly Journal of Mathematics. Oxford. Second Series, vol. 11, pp. 36-48, 1940.

[17] S. Roscani and E. Santillan Marcus, “Two equivalent Stefan's problems for the time-fractional diffusion equation," Fractional Calculus and Applied Analysis, vol. 16, no. 4, pp. 802-815, 2013.

[18] J. Liu and M. Xu, "Some exact solutions to Stefan problems with fractional differential equations," Journal of Mathematical Analysis and Applications, vol. 351, no. 2, pp. 536-542, 2009.

[19] S. D. Eidelman, S. D. Ivasyshen, and A. N. Kochubei, Analytic Methods in the Theory of Differential and Pseudo-Differential Equations of Parabolic Type, Birkhäuser, 2004.

[20] R. Gorenflo, Y. Luchko, and F. Mainardi, "Analytical properties and applications of the Wright function," Fractional Calculus \& Applied Analysis, vol. 2, no. 4, pp. 383-414, 1999.

[21] H. Brézis, Análisis Funcional Teoría y aplicaciones. Versión española de Juan Ramón Esteban, Alianza Editorial, Madrid, Spain, 1984.

[22] F. Mainardi, "On the initial value problem for the fractional diffusion-wave equation," in Proceeding of VIIth International Conference on Waves and Stability in Continuous Media WASCOM, Bologna, Italy, 4-7 October 1993, S. Rionero and T. Ruggeri, Eds., pp. 246-251, World Scientific, Singapore, 1994.

[23] Y. Lin and C. Xu, "Finite difference/spectral approximations for the time-fractional diffusion equation," Journal of Computational Physics, vol. 225, no. 2, pp. 1533-1552, 2007.

[24] W. Chen, L. Ye, and H. Sun, "Fractional diffusion equations by the Kansa method," Computers \& Mathematics with Applications, vol. 59, no. 5, pp. 1614-1620, 2010.

[25] M. Javidi and B. Ahmad, "Numerical solution of fractional partial differential equations by numerical Laplace inversion technique," Advances in Difference Equations, vol. 2013, article $375,2013$. 


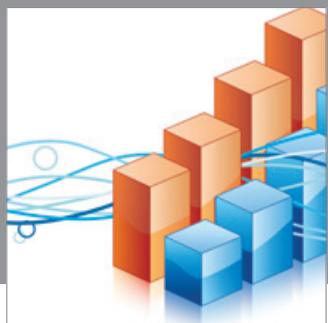

Advances in

Operations Research

mansans

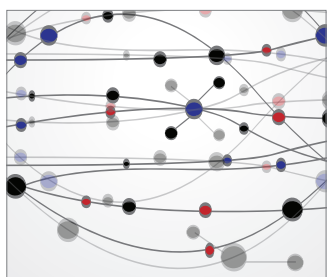

The Scientific World Journal
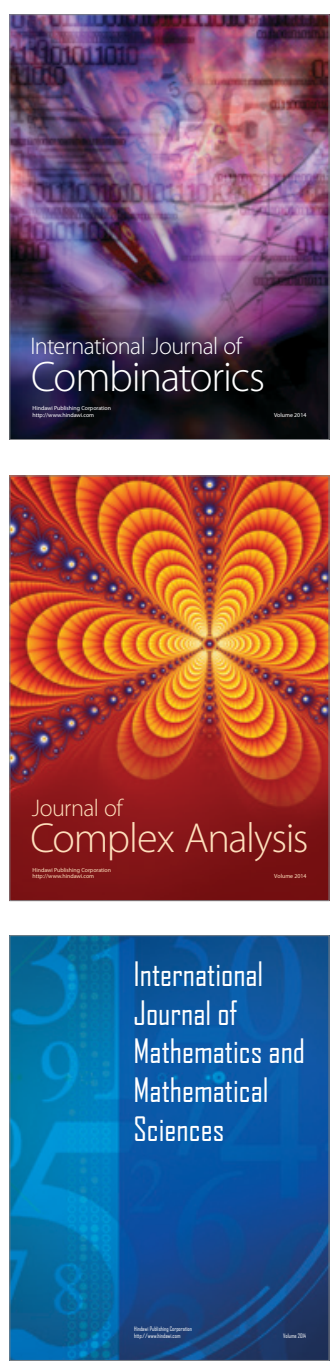
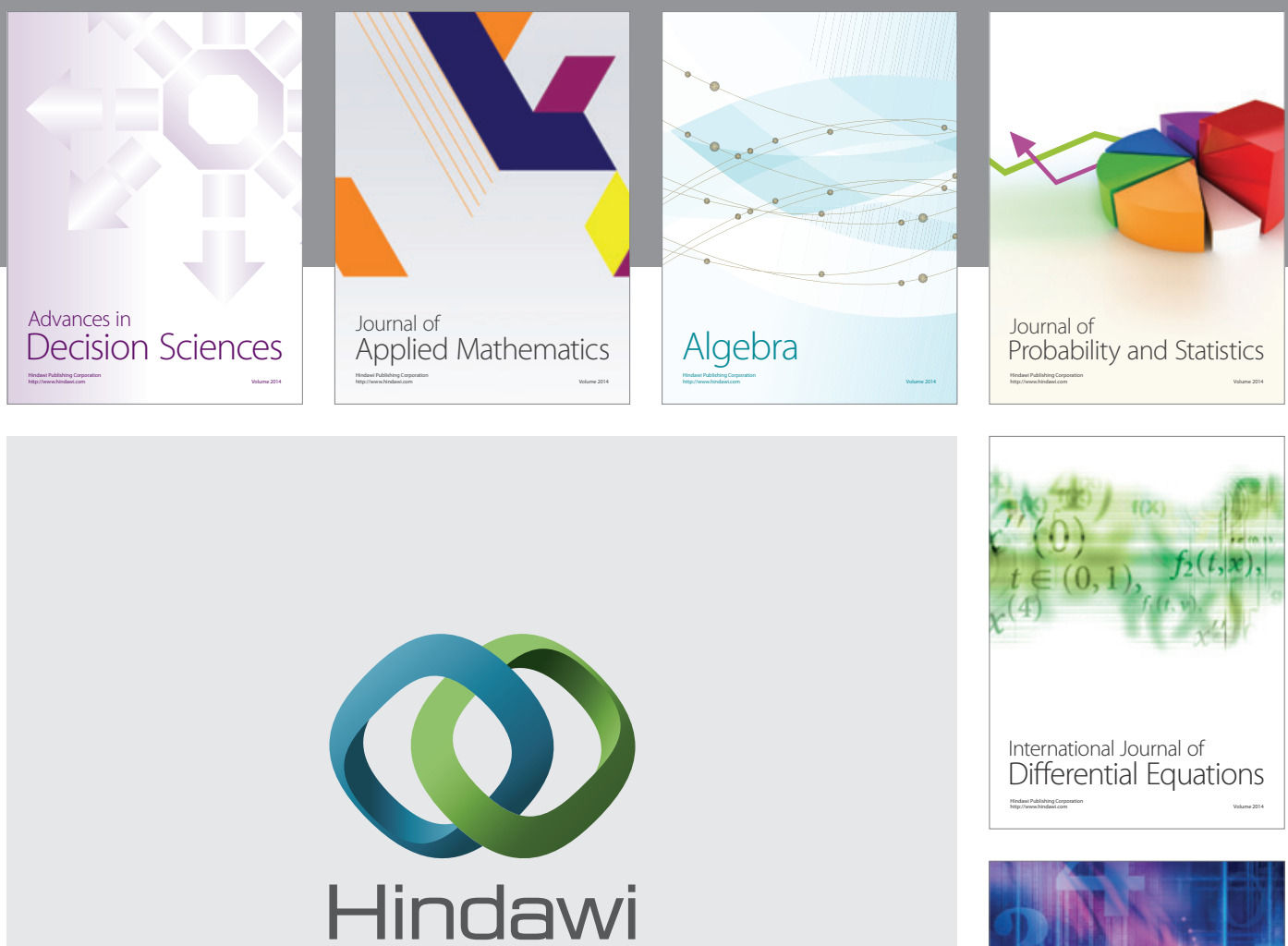

Submit your manuscripts at http://www.hindawi.com
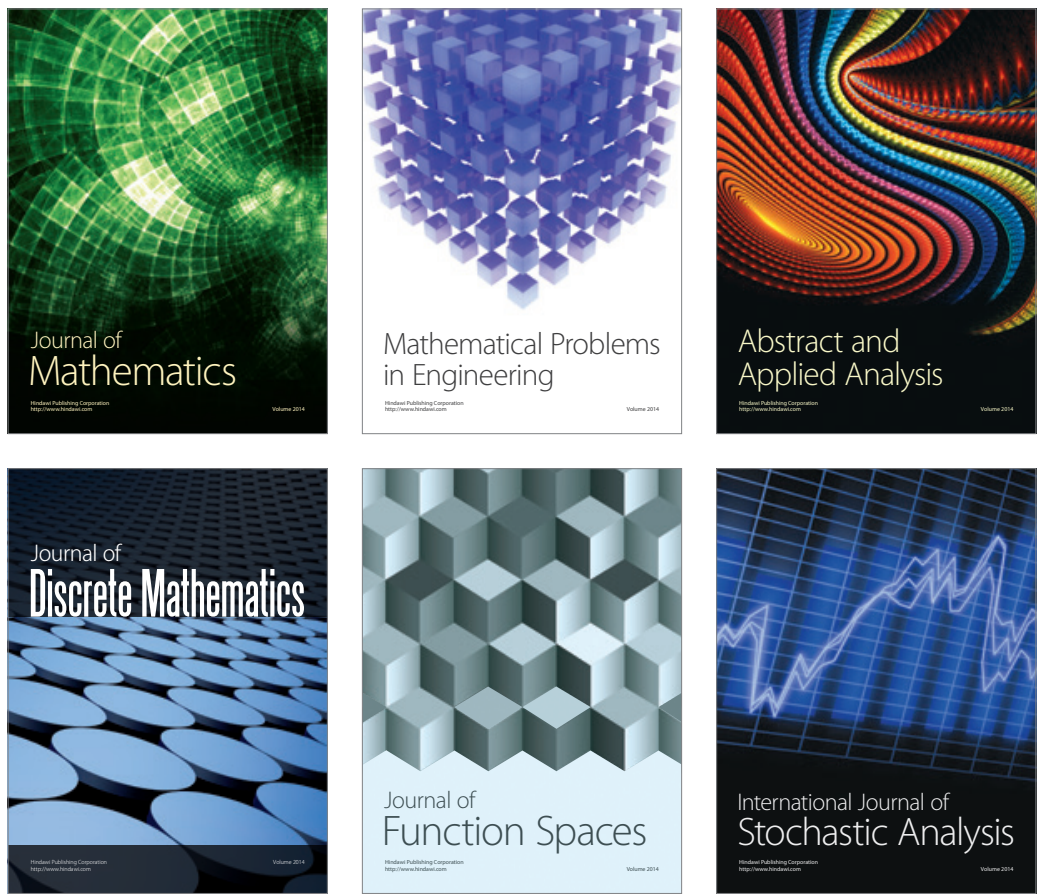

Journal of

Function Spaces

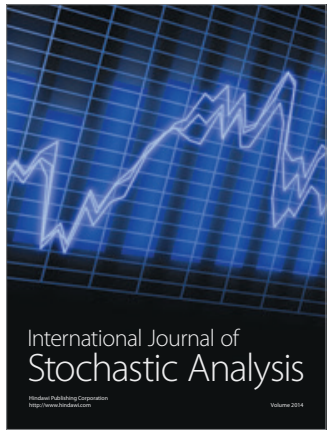

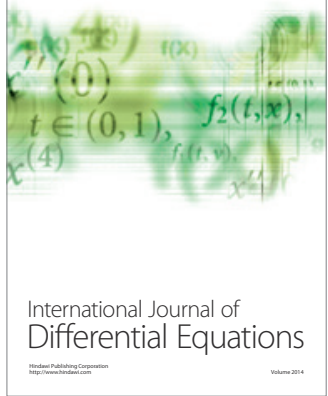
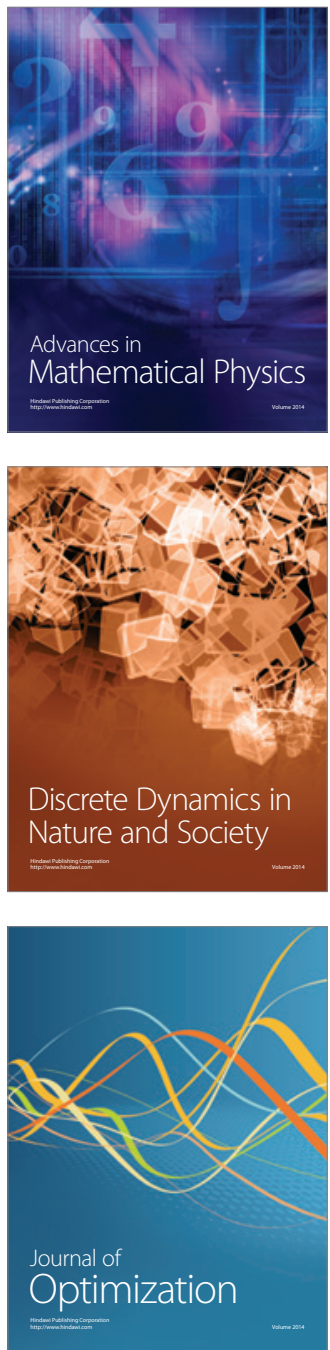